\title{
Linear Prediction Approach for Efficient Frequency Estimation of Multiple Real Sinusoids: Algorithms and Analyses
}

\author{
H. C. So, Member, IEEE, Kit Wing Chan, Y. T. Chan, Senior Member, IEEE, and K. C. Ho, Senior Member, IEEE
}

\begin{abstract}
Based on the linear prediction property of sinusoidal signals, two constrained weighted least squares frequency estimators for multiple real sinusoids embedded in white noise are proposed. In order to achieve accurate frequency estimation, the first algorithm uses a generalized unit-norm constraint, while the second method employs a monic constraint. The weighting matrices in both methods are a function of the frequency parameters and are obtained in an iterative manner. For the case of a single real tone with sufficiently large data samples, both estimators provide nearly identical frequency estimates and their performance approaches Cramér-Rao lower bound (CRLB) for white Gaussian noise before the threshold effect occurs. Algorithms for closed-form single-tone frequency estimation are also devised. Computer simulations are included to corroborate the theoretical development and to contrast the estimator performance with the CRLB for different frequencies, observation lengths and signal-to-noise ratio (SNR) conditions.
\end{abstract}

Index Terms-Frequency estimation, linear prediction, monic constraint, real sinusoids, unit-norm constraint, weighted least squares.

\section{INTRODUCTION}

$\mathbf{T}$ HE problem of estimating the frequencies of sinusoidal components from a finite number of noisy discrete-time measurements has attracted a great deal of attention [1]-[6] and still is an active research area to date, because of its wide applications in science and engineering.

In spite of the fact that there are numerous frequency estimation schemes in the literature, they can be generally classified as nonparametric or parametric approaches. The nonparametric frequency estimators, including the periodogram and correlogram methods, are based directly on the Fourier transform. Although no assumptions are made about the observed data sequence, the resolution, or ability to resolve closely spaced frequencies using the nonparametric approach is fundamentally limited by the length of the data available. Alternatively, the

\footnotetext{
Manuscript received October 3, 2003; revised July 12, 2004. The associate editor coordinating the review of this manuscript and approving it for publication was Dr. Franz Hlawatsch.

H. C. So and K. W. Chan are with Department of Computer Engineering and Information Technology, City University of Hong Kong, Hong Kong (e-mail: ithcso@cityu.edu.hk; k.w.chan@student.cityu.edu.hk).

Y. T. Chan is with Department of Electrical and Computer Engineering, Royal Military College of Canada, Kingston, ON, K7K 7B4 Canada (e-mail: chan-yt@ rmc.ca).

K. C. Ho is with Department of Electrical and Computer Engineering, University of Missouri-Columbia, Columbia, MO 65211 USA (e-mail: hod@ missouri.edu).

Digital Object Identifier 10.1109/TSP.2005.849154
}

parametric approach, which assumes that the signal satisfies a generating model with known functional form, can attain a higher resolution. Well-known parametric frequency estimation techniques include maximum likelihood (ML) [7], nonlinear least squares (NLS) [8], Prony's method, instrumental variable (IV) [9], Yule-Walker equations [10], total least squares [11], iterative filtering [12] and subspace methods such as truncated singular value decomposition, MUSIC and ESPRIT [4]. In fact, under additive white Gaussian noise, the ML and NLS methods are equivalent and both are statistically efficient in the sense that the estimator variances achieve Cramér-Rao lower bound (CRLB) asymptotically, but their computational requirements are extremely demanding. On the other hand, the rest of the above mentioned parametric techniques utilize the linear prediction (LP) property of sinusoidal signals, and generally speaking, they provide suboptimum estimation performance but are computationally efficient.

The motivation of this paper is to investigate if the LP approach can attain optimum frequency estimation performance for multiple real sinusoids. In our previous work [13], a simple frequency estimator for a single real tone is devised based on the framework of Pisarenko [14], who was the first to exploit the eigenstructure of the covariance matrix in frequency estimation. Although it is an improvement to the original Pisarenko harmonic decomposition (PHD) method, the algorithm does not give efficient estimates and cannot be extended to multiple frequency estimation. The major contributions of this paper can be summarized as i) two efficient LP-based frequency estimators for multiple real sinusoids are devised and ii) a proof for their optimality in the case of a single real tone embedded in white Gaussian noise is provided, that is, we have shown that the variances of the frequency estimates can approach the CRLB when the data length is sufficiently large.

The rest of the paper is organized as follows. In Section II, the problem of multiple frequency estimation is formulated and the LP property of sinusoidal signals is reviewed. In Section III, we first develop a constrained least squares (LS) frequency estimator which can be considered as a reformulation of Pisarenko's method, and then it is improved through the technique of weighted least squares (WLS) with a generalized unit-norm constraint. The WLS frequency estimator with monic constraint is devised in Section IV. Section V studies the relationship between the two estimators as well as their biases and variances for a single real tone when there are infinite data samples. Closed-form single-tone frequency estimation is investigated in Section VI. Numerical examples are presented 
in Section VII to corroborate the analytical development and to evaluate the performance of the proposed algorithms by comparing with the CRLB. Finally, conclusions are drawn in Section VIII.

\section{PROBlem Formulation AND LineAR PREDICTION PROPERTY}

The problem of multiple real sinusoidal frequency estimation is formulated as follows. Given the discrete-time noisy measurements

$$
x(n)=s(n)+q(n), \quad n=0,1, \ldots, N-1
$$

where

$$
s(n)=\sum_{l=1}^{L} \alpha_{l} \sin \left(\omega_{l} n+\phi_{l}\right), \quad l=1,2, \ldots, L
$$

with $\alpha_{l}, \omega_{l} \in(0, \pi)$ and $\phi_{l} \in[0,2 \pi)$ are unknown constants which denote the amplitude, frequency and phase of the $l$-th real-valued sinusoid, respectively, while $q(n)$ is an additive white noise with unknown variance $\sigma_{q}^{2}$. For frequency estimation in colored noise, the reader is referred to [15]-[19]. Furthermore, it is assumed that the number of sinusoids, $L$, is known a priori. The task here is to find $\omega_{l}, l=1,2, \ldots, L$, from the $N$ samples of $x(n)$.

It is well known that $s(n)$ can be uniquely expressed as a linear combination of its previous $2 L$ samples as

$$
s(n)=-\sum_{i=1}^{2 L} a_{i} s(n-i)
$$

where $\left\{a_{i}\right\}$ are referred to as the LP coefficients. A relationship between $\left\{\omega_{l}\right\}$ and $\left\{a_{i}\right\}$ is given by [9]

$$
\sum_{i=0}^{2 L} a_{i} \exp \left(-j \omega_{l} i\right)=0
$$

where $a_{0}=1$ and $a_{i}=a_{2 L-i}, i=0,1, \ldots, L$, are real, which implies that the $L$ frequencies are equal to the phases of the roots of $\sum_{i=0}^{2 L} a_{i} z^{i}=0$, namely, $\left\{\exp \left( \pm j \omega_{l}\right)\right\}$. In our LP approach, we first estimate $\left\{a_{i}\right\}$ and the frequency estimates are then determined using (4).

\section{WEIGHTED LEAST SQUARES FREQUENCY ESTIMATION With GENERALIZED UNIT-NORM CONSTRAINT}

Based on the LP property of sinusoidal signals and the symmetry of $\left\{a_{i}\right\}$, we define an error function of the form

$$
e(n)=\sum_{i=0}^{L-1} \tilde{a}_{i}[x(n-i)+x(n-2 L+i)]+\tilde{a}_{L} x(n-L)
$$

where $\left\{\tilde{a}_{i}\right\}$ are the optimization variables of $\left\{a_{i}\right\}$ up to a scalar since $\tilde{a}_{0}$ is not fixed to be unity. The corresponding LS cost function is simply

$$
J(\tilde{\mathbf{a}})=\sum_{n=2 L}^{N-1} e^{2}(n)=\mathbf{e}^{T} \cdot \mathbf{e}=\tilde{\mathbf{a}}^{T} \cdot \mathbf{X}^{T} \mathbf{X} \cdot \tilde{\mathbf{a}}
$$

where we have (7)-(9), shown at the bottom of the page, and $T$ represents the transpose operation. Decomposing $\mathbf{X}$ into signal and noise components, we have

$$
\mathbf{X}=\mathbf{S}+\mathbf{Q}
$$

where the expressions of $\mathbf{S}$ and $\mathbf{Q}$ are shown in the equation at the bottom of this page and in the first equation at the bottom of the next page, respectively. By substituting (10) into (6) and then taking the expected value, it is easy to show that

$$
E\{J(\tilde{\mathbf{a}})\}=(\mathbf{S} \cdot \tilde{\mathbf{a}})^{T} \cdot \mathbf{S} \cdot \tilde{\mathbf{a}}+(N-2 L) \cdot \tilde{\mathbf{a}}^{T} \cdot \boldsymbol{\Sigma} \cdot \tilde{\mathbf{a}} \cdot \sigma_{q}^{2}
$$

where $\boldsymbol{\Sigma}=\operatorname{diag}(2,2, \ldots, 2,1)$. Note that if $\tilde{\mathbf{a}}=\mathbf{a} \tilde{a}_{i}=a_{i}$, $i=0,1, \ldots, L$, then $\mathbf{S} \cdot \mathbf{a}$ is a zero vector. Since the second term of (11) is a function of $\tilde{\mathbf{a}}$, we see that the minimum of $E\{J(\tilde{\mathbf{a}})\}$ does not correspond to the desired a except when $q(n)=0$ only. As a result, biased frequency estimates will be obtained via minimizing (6) in the presence of noise. Similar to the unit-norm constraint approach [20], [21], a straightforward way to get rid of the frequency bias and avoid the trivial solution of all zeros is

$$
\begin{aligned}
& \mathbf{e}=[e(N-1), e(N-2), \ldots, e(2 L)]^{T} \\
& \mathbf{X}=\left[\begin{array}{cccc}
x(N-1)+x(N-2 L-1) & \cdots & x(N-L)+x(N-L-2) & x(N-L-1) \\
x(N-2)+x(N-2 L-2) & \cdots & x(N-L-1)+x(N-L-3) & x(N-L-2) \\
\vdots & \vdots & \vdots & \vdots \\
x(2 L)+x(0) & \cdots & x(L+1)+x(L-1) & x(L)
\end{array}\right] \\
& \tilde{\mathbf{a}}=\left[\tilde{a}_{0}, \tilde{a}_{1}, \ldots, \tilde{a}_{L}\right]^{T}
\end{aligned}
$$

$$
\mathbf{S}=\left[\begin{array}{cccc}
s(N-1)+s(N-2 L-1) & \cdots & s(N-L)+s(N-L-2) & s(N-L-1) \\
s(N-2)+s(N-2 L-2) & \cdots & s(N-L-1)+s(N-L-3) & s(N-L-2) \\
\vdots & \vdots & \vdots & \vdots \\
s(2 L)+s(0) & \cdots & s(L+1)+s(L-1) & s(L)
\end{array}\right]
$$


to minimize $J(\tilde{\mathbf{a}})$ subject to a generalized unit-norm constraint of the form

$$
\tilde{\mathbf{a}}^{T} \cdot \mathbf{\Sigma} \cdot \tilde{\mathbf{a}}=1
$$

This formulation is not really novel because it only replaces the sample covariance matrix in the Pisarenko's method [14] by the data matrix $\mathbf{X}^{T} \mathbf{X}$ and utilizes $\tilde{a}_{i}=\tilde{a}_{2 L-i}, l=0,1, \ldots, L$. Using the method of Lagrange multipliers, the problem of minimizing (6) subject to (12) can be solved as follows. Define the Lagrangian

$$
\mathcal{L}(\tilde{\mathbf{a}}, \lambda)=\tilde{\mathbf{a}}^{T} \cdot \mathbf{X}^{T} \mathbf{X} \cdot \tilde{\mathbf{a}}+\lambda\left(1-\tilde{\mathbf{a}}^{T} \cdot \boldsymbol{\Sigma} \cdot \tilde{\mathbf{a}}\right)
$$

where $\lambda$ is the Lagrange multiplier. Differentiating $\mathcal{L}(\tilde{\mathbf{a}}, \lambda)$ with respect to $\tilde{\mathbf{a}}$ and then setting the resultant expression to zero yields

$$
\frac{\partial \mathcal{L}(\tilde{\mathbf{a}}, \lambda)}{\partial \tilde{\mathbf{a}}}=2 \mathbf{X}^{T} \mathbf{X} \cdot \hat{\mathbf{a}}-2 \lambda \boldsymbol{\Sigma} \cdot \hat{\mathbf{a}}=0 \Rightarrow \mathbf{X}^{T} \mathbf{X} \cdot \hat{\mathbf{a}}=\lambda \boldsymbol{\Sigma} \cdot \hat{\mathbf{a}}
$$

where $\hat{\mathbf{a}}=\left[\hat{a}_{0}, \hat{a}_{1}, \ldots, \hat{a}_{L}\right]^{T}$ is the estimate of $\left\{a_{i}\right\}$ up to a scalar, and $\hat{\mathbf{a}}$ is given by [22] the generalized eigenvector corresponding to the smallest generalized eigenvalue of $\left(\mathbf{X}^{T} \mathbf{X}\right.$, $\boldsymbol{\Sigma})$. Note that since $\boldsymbol{\Sigma}$ is diagonal, the generalized eigenvalue problem can be easily converted into a standard eigenvalue problem by finding the eigenvector corresponding to the smallest eigenvalue of $\boldsymbol{\Sigma}^{-1 / 2} \cdot \mathbf{X}^{T} \mathbf{X} \cdot \boldsymbol{\Sigma}^{-1 / 2}$. Alternatively, the constrained optimization problem in (6) and (12) is also equivalent to minimizing an unconstrained cost function [21]:

$$
\frac{\tilde{\mathbf{a}}^{T} \cdot \mathbf{X}^{T} \mathbf{X} \cdot \tilde{\mathbf{a}}}{\tilde{\mathbf{a}}^{T} \cdot \mathbf{\Sigma} \cdot \tilde{\mathbf{a}}}
$$

Since the estimator based on (14) or (15) is closely related to the PHD method, we refer it to as reformed Pisarenko harmonic decomposer (RPHD). In fact, we have derived a closed-form solution for the RPHD when $L=1$ by setting $\hat{a}_{0}=\hat{a}_{2}=1$ in (15), and the estimate of $a_{1}$ is given by [13]

$$
\hat{a}_{1}=-\frac{B_{N}+\sqrt{B_{N}^{2}+8 A_{N}^{2}}}{2 A_{N}}
$$

where $A_{N}=\sum_{n=2}^{N-1}[x(n)+x(n-2)] x(n-1)$ and

$B_{N}=x^{2}(N-1)-x^{2}(N-2)-x^{2}(1)+x^{2}(0)+2 \sum_{n=2}^{N-1} x(n) x(n-2)$.

The frequency estimate is then computed as $\cos ^{-1}\left(-\hat{a}_{1} / 2\right)$. For single-tone frequency estimation, using (14) or (15) is equivalent to employing (16), although the latter has an obvious advantage of much smaller computational requirement, particularly when $N$ is large. It is demonstrated [13] that the estimator of (16) outperforms the original Pisarenko's method for small $N$ or large signal-to-noise ratio (SNR), which can be explained by the fact that optimum sample covariance matrix estimation is difficult to achieve in such conditions, but it is not optimum as it has variances of order $N^{-2}$ or $O\left(N^{-2}\right)$ and $O\left(N^{-1}\right)$ at high and low SNRs, respectively, for $N \rightarrow \infty$. We expect that these findings also hold for multiple frequency estimation based on (14) or (15).

Since LS optimization generally cannot provide the best solution [23], this gives an alternative explanation for the suboptimality of Pisarenko's method as well as the RPHD. From (6), an improvement to the RPHD is to add a symmetric weighting matrix, say, $\mathbf{W}$, to the LS cost function. A typical choice of $\mathbf{W}$ is [23]:

$$
\mathbf{W}=\left(E\left\{\mathbf{e}_{\mathbf{q}} \cdot \mathbf{e}_{\mathbf{q}}^{T}\right\}\right)^{-1}
$$

where $\mathbf{e}_{\mathbf{q}}=\left[e_{q}(N-1), e_{q}(N-2), \ldots, e_{q}(2 L)\right]^{T}$ with $e_{q}(n)=$ $\sum_{i=0}^{L-1} a_{i}[q(n-i)+q(n-2 L+i)]+a_{L} q(n-L)$ is the residual error at the true $\left\{a_{i}\right\}$. The inverse of $\mathbf{W}$ is calculated as (18), shown at the bottom of the page.

Now, the constraint of (12) will be revised as the cost function is modified, and the development is given as follows. From (6) and (10), we expand e as

$$
\mathbf{e}=\mathbf{S} \cdot \tilde{\mathbf{a}}+\mathbf{Q} \cdot \tilde{\mathbf{a}} \text {. }
$$

$$
\mathbf{Q}=\left[\begin{array}{cccc}
q(N-1)+q(N-2 L-1) & \cdots & q(N-L)+q(N-L-2) & q(N-L-1) \\
q(N-2)+q(N-2 L-2) & \cdots & q(N-L-1)+q(N-L-3) & q(N-L-2) \\
\vdots & \vdots & \vdots & \vdots \\
q(2 L)+q(0) & \cdots & q(L+1)+q(L-1) & q(L)
\end{array}\right]
$$

$$
\mathbf{W}^{-1}=\left[\begin{array}{ccccccc}
a_{L}^{2}+2 \sum_{i=0}^{L-1} a_{i}^{2} & 2 \sum_{i=0}^{L-1} a_{i} a_{i+1} & \cdots & a_{0}^{2} & 0 & \cdots & 0 \\
2 \sum_{i=0}^{L-1} a_{i} a_{i+1} & a_{L}^{2}+2 \sum_{i=0}^{L-1} a_{i}^{2} & 2 \sum_{i=0}^{L-1} a_{i} a_{i+1} & \cdots & a_{0}^{2} & \cdots & 0 \\
\vdots & \vdots & \vdots & \vdots & \vdots & \vdots & \vdots \\
0 & \cdots & 0 & \cdots & \cdots & 2 \sum_{i=0}^{L-1} a_{i} a_{i+1} & a_{L}^{2}+2 \sum_{i=0}^{L-1} a_{i}^{2}
\end{array}\right] \sigma_{q}^{2} .
$$


Premultiplying e by $\mathbf{W}$ gives

$$
\mathbf{W} \cdot \mathbf{e}=\mathbf{W} \cdot \mathbf{S} \cdot \tilde{\mathbf{a}}+\mathbf{W} \cdot \mathbf{Q} \cdot \tilde{\mathbf{a}} .
$$

Premultiplying (20) by $\mathbf{e}^{T}$ and then taking the expected value, we get

$$
E\left\{\mathbf{e}^{T} \cdot \mathbf{W} \cdot \mathbf{e}\right\}=(\mathbf{S} \cdot \tilde{\mathbf{a}})^{T} \cdot \mathbf{W} \cdot \mathbf{S} \cdot \tilde{\mathbf{a}}+\tilde{\mathbf{a}}^{T} \cdot \Upsilon \mathbf{\Upsilon} \cdot \tilde{\mathbf{a}} \cdot \sigma_{q}^{2}
$$

where

$$
\Upsilon=\frac{E\left\{\mathbf{Q}^{T} \cdot \mathbf{W} \cdot \mathbf{Q}\right\}}{\sigma_{q}^{2}}
$$

The matrix $\Upsilon$ is computed as (see Appendix A) (23), shown at the bottom of the page, where $D_{j}=\sum_{i=1}^{N-2 L-j}[\mathbf{W}]_{i, i+j}$, $j=0,1, \ldots, 2 L$, with $[\mathbf{W}]_{i, j}$ represents the $(i, j)$ th element of W.

As a result, the LP-based WLS frequency estimation with the generalized unit-norm constraint is achieved via minimizing

$$
J_{1}(\tilde{\mathbf{a}})=\mathbf{e}^{T} \cdot \mathbf{W} \cdot \mathbf{e}=\tilde{\mathbf{a}}^{T} \cdot \mathbf{X}^{T} \cdot \mathbf{W} \cdot \mathbf{X} \cdot \tilde{\mathbf{a}}
$$

subject to the constraint of

$$
\tilde{\mathbf{a}}^{T} \cdot \Upsilon \cdot \tilde{\mathbf{a}}=1
$$

Following (13) and (14), the frequency estimates are determined from another generalized eigenvalue problem:

$$
\mathbf{X}^{T} \cdot \mathbf{W} \cdot \mathbf{X} \cdot \hat{\mathbf{a}}=\lambda \mathbf{\Upsilon} \cdot \hat{\mathbf{a}} .
$$

It is observed that (14) is only a special case of (26) when $\mathbf{W}$ is an identity matrix and $\boldsymbol{\Upsilon}=\boldsymbol{\Sigma}$.

Since a is not available, we do not have the exact form of $\mathbf{W}$. In actual implementation, we can first set $\mathbf{W}$ to the identity matrix to obtain an initial estimate $\hat{\mathbf{a}}$. This initial solution will be used to obtain a scaled estimate of $\mathbf{W}$, denoted by $\hat{\mathbf{W}}$, where we replace a by $\hat{\mathbf{a}}$ in $\mathbf{W}^{-1}$ and then divide it by $\sigma_{q}^{2}$ so that $\hat{\mathbf{W}}$ is characterized by â only. The process is then iterated to obtain a better solution. The detailed algorithm is given as follows.

i) Find an initial estimate â by the RPHD via computing the eigenvector corresponding to the minimum eigenvalue of $\boldsymbol{\Sigma}^{-1 / 2} \cdot \mathbf{X}^{T} \mathbf{X} \cdot \boldsymbol{\Sigma}^{-1 / 2}$, or by other high resolution frequency estimation techniques. ii) Use the estimated â to construct $\hat{\mathbf{W}}^{-1}$ and then take the inverse to obtain $\hat{\mathbf{W}}$ as well as $\boldsymbol{\Upsilon}$.

iii) Compute the generalized eigenvector corresponding to the minimum generalized eigenvalue of $\left(\mathbf{X}^{T} \cdot \hat{\mathbf{W}} \cdot \mathbf{X}\right.$, $\Upsilon)$.

iv) Repeat Steps ii) and iii) until a reliable solution has been reached.

v) Use the finalized â to compute the frequency estimates.

Although we are not aware [24] of the existence of a proof of global convergence for the above relaxation algorithm, simulation results in Section VII demonstrate that using a few iterations in the estimation procedure, the algorithm is able to achieve global convergence with performance approaching the CRLB for sufficiently large SNRs and/or data lengths. Since normally we have $N \gg L$, the major computational requirement of the algorithm is to determine the inverse of $\hat{\mathbf{W}}^{-1}$, which requires $O\left(N^{3}\right)$ multiplications if its special structure is ignored. Although we have not addressed its fast realization, efficient algorithms for symmetric sparse Toeplitz matrix inverse are found in the scientific computing literature, and interested readers can refer to works in this field. As a final remark, an advantage of our approach over commonly used high-resolution methods such as MUSIC and ESPRIT [4], [5] is that we do not require a user-chosen parameter which corresponds to the size of the data matrix or sample covariance matrix. Nevertheless, we need to specify the stopping criterion, which is in terms of number of iterations in our study, for the proposed approach.

\section{Weighted Least SQuares Frequency Estimation With MONIC CONSTRAINT}

Apart from the unit-norm constraint, monic constraint is also commonly employed in the literature [21], [25], [26]. In this Section, we will derive the WLS frequency estimator with monic constraint based on (24). With the constraint of $\tilde{a}_{0}=1$, we partition $\tilde{\mathbf{a}}$ as

$$
\tilde{\mathbf{a}}=\left[\begin{array}{ll}
1 & \tilde{\boldsymbol{\rho}}^{T}
\end{array}\right]^{T}
$$

where

$$
\tilde{\boldsymbol{\rho}}=\left[\tilde{a}_{1}, \tilde{a}_{2}, \ldots, \tilde{a}_{L}\right]^{T} .
$$

The data matrix is then partitioned accordingly as

$$
\mathbf{X}=\left[\begin{array}{ll}
\mathbf{X}_{1} & \mathbf{X}_{\mathbf{2}}
\end{array}\right]
$$


where

$$
\mathbf{X}_{\mathbf{1}}=\left[\begin{array}{c}
x(N-1)+x(N-2 L-1) \\
x(N-2)+x(N-2 L-2) \\
\vdots \\
x(2 L)+x(0)
\end{array}\right]
$$

and the expression of $\mathbf{X}_{\mathbf{2}}$ is shown at the bottom of the page. Substituting (27) and (29) into (24), the monic constraint WLS frequency estimator is to minimize

$$
J_{2}(\tilde{\boldsymbol{\rho}})=\left(\mathbf{X}_{\mathbf{1}}+\mathbf{X}_{\mathbf{2}} \cdot \tilde{\boldsymbol{\rho}}\right)^{T} \cdot \mathbf{W} \cdot\left(\mathbf{X}_{\mathbf{1}}+\mathbf{X}_{\mathbf{2}} \cdot \tilde{\boldsymbol{\rho}}\right) .
$$

Differentiating $J_{2}(\tilde{\boldsymbol{\rho}})$ with respect to $\tilde{\boldsymbol{\rho}}$ and then setting the resultant expression to zero yields

$$
\hat{\boldsymbol{\rho}}=-\left(\mathbf{X}_{\mathbf{2}}^{T} \cdot \mathbf{W} \cdot \mathbf{X}_{\mathbf{2}}\right)^{-1} \cdot \mathbf{X}_{\mathbf{2}}^{T} \cdot \mathbf{W} \cdot \mathbf{X}_{\mathbf{1}}
$$

where $\hat{\boldsymbol{\rho}}$ is the estimate of $\left[a_{1}, a_{2}, \ldots, a_{L}\right]^{T}$. At first sight, (31) seems to give biased estimation because $\mathbf{X}_{\mathbf{1}}$ is correlated with $\mathbf{X}_{\mathbf{2}}$ [23]. In fact, it is true that (31) will provide biased frequency estimates when standard LS is employed, that is, when $\mathbf{W}$ is replaced by an identity matrix. However, in the next Section, we argue that the bias of the solution in (31) is insignificant for a sufficiently large observation length, due to the presence of our particular choice of weighting matrix W. As in the unitnorm constraint frequency estimator, $\hat{\mathbf{W}}$ is employed instead in practice. We summarize the iterative procedure for the LP-based WLS frequency estimation with monic constraint as follows.

i) Find an initial estimate $\hat{\mathbf{a}}$ based on the eigenvector corresponding to the minimum eigenvalue of $\Sigma^{-1 / 2} \cdot \mathbf{X}^{T} \mathbf{X}$. $\boldsymbol{\Sigma}^{-1 / 2}$, and then normalize the first element of $\hat{\mathbf{a}}$ to get $\hat{\boldsymbol{\rho}}$, or otherwise.

ii) Use the estimated $\hat{\boldsymbol{\rho}}$ to construct $\hat{\mathbf{W}}^{-1}$ and then take the inverse to obtain $\mathbf{W}$.

iii) Compute an improved $\hat{\boldsymbol{\rho}}$ using (31).

iv) Repeat Steps ii) and iii) until a reliable solution has been reached.

v) Use the finalized $\hat{\boldsymbol{\rho}}$ to compute the frequency estimates.

It is noteworthy that the above algorithm is similar to the iterative quadratic maximum likelihood (IQML) approach [27], [28]. However, their formulations are quite different as the IQML estimate is derived from the ML criterion while ours is based on the WLS technique.

\section{RELATIONSHIP BETWEEN ESTIMATORS AND PERFORMANCE ANALYSIS}

We will first show that the WLS frequency estimates using the generalized unit-norm and monic constraints are very close, and then derive the estimator bias and variance. In order to make the theoretical development in this Section feasible, we limit to the case of a single sinusoid because the analysis for $L>1$ will be extremely complicated. To proceed, the following assumptions are required:

A1): The ideal weighting matrix $\mathbf{W}$ is employed in both frequency estimators. This assumption is used to simplify the derivation. Although $\mathbf{W}$ generally differs from its estimate $\hat{\mathbf{W}}$, Section VII indicates that the simulation and theoretical results agree quite well for sufficiently large $N$ and/or SNR, although the analysis is based on $\mathbf{W}$.

A2): The number of measurement samples is sufficiently large. A major difficulty in developing the theoretical study is that there is no closed-form expression for $\mathbf{W}$, even for $L=1$. Through considering $N \rightarrow \infty$, we are able to find a closed-form expression to approximate $\mathbf{W}$. This assumption also implies the error in $\hat{\mathbf{a}}$, and, hence, $\hat{\mathbf{W}}$, is very small.

For $L=1$, we redefine $\Upsilon$ accordingly with the use of (22) and (23):

$$
\Upsilon=E\left\{\mathbf{Q}^{T} \cdot \mathbf{W} \cdot \mathbf{Q}\right\}=\left[\begin{array}{cc}
2\left(D_{0}+D_{2}\right) & 2 D_{1} \\
2 D_{1} & D_{0}
\end{array}\right]
$$

The next step is to express $\Upsilon$ in terms of $a_{1}=-2 \cos \left(\omega_{1}\right)$, which is achieved via the development of an approximate form for W (See Appendix B):

$$
\begin{aligned}
\mathbf{W} & =\left[\begin{array}{ccccccc}
2+a_{1}^{2} & 2 a_{1} & 1 & 0 & \ldots & 0 & 0 \\
2 a_{1} & 2+a_{1}^{2} & 2 a_{1} & 1 & \ldots & 0 & 0 \\
\vdots & \vdots & \vdots & \vdots & \vdots & \vdots & \\
0 & 0 & 0 & 0 & \ldots & 2+a_{1}^{2} & 2 a_{1} \\
0 & 0 & 0 & 0 & \ldots & 2 a_{1} & 2+a_{1}^{2}
\end{array}\right] \cdot \sigma_{q}^{-2} \\
& \approx \mathbf{U}^{2} \cdot \sigma_{q}^{-2}
\end{aligned}
$$

where the approximation error decreases as $N$ increases, and we have (34), shown at the bottom of the next page. Note that from (33) and (34), we can use an estimate of $\mathbf{U}^{2}$ instead of $\mathbf{W}$ in the algorithms for sufficiently large $N$, but there is no obvious advantage because the computational complexity of $\mathbf{U}^{2}$ is also $O\left(N^{3}\right)$. Substituting (33) and (34) into (32), we obtain (see Appendix C):

$$
\Upsilon \approx D_{0}\left[\begin{array}{cc}
2\left(1+\cos \left(2 \omega_{1}\right)\right) & 2 \cos \left(\omega_{1}\right) \\
2 \cos \left(\omega_{1}\right) & 1
\end{array}\right]
$$

$$
\mathbf{X}_{\mathbf{2}}=\left[\begin{array}{cccc}
x(N-2)+x(N-2 L) & \cdots & x(N-L)+x(N-L-2) & x(N-L-1) \\
x(N-3)+x(N-2 L-1) & \cdots & x(N-L-1)+x(N-L-3) & x(N-L-2) \\
\vdots & \vdots & \vdots & \vdots \\
x(2 L-1)+x(1) & \cdots & x(L+1)+x(L-1) & x(L)
\end{array}\right]
$$


On the other hand, premultiplying both sides of (26) by $\hat{\mathbf{a}}^{T}$ and with the use of (25), the minimum generalized eigenvalue of $\left(\mathbf{X}^{T} \cdot \mathbf{W} \cdot \mathbf{X}, \Upsilon\right)$ can be expressed as

$$
\lambda=\hat{\mathbf{a}}^{T} \cdot \mathbf{X}^{T} \cdot \mathbf{W} \cdot \mathbf{X} \cdot \hat{\mathbf{a}}=\hat{\mathbf{a}}^{T} \cdot(\mathbf{S}+\mathbf{Q})^{T} \cdot \mathbf{W} \cdot(\mathbf{S}+\mathbf{Q}) \cdot \hat{\mathbf{a}} .
$$

As â is getting closer to $\left[1, a_{1}\right]^{T}$ and considering $N$ tends to infinity such that $\mathbf{Q}^{T} \cdot \mathbf{W} \cdot \mathbf{Q}$ approaches $E\left\{\mathbf{Q}^{T} \cdot \mathbf{W} \cdot \mathbf{Q}\right\}$ and the terms involving both $\mathbf{S}$ and $\mathbf{Q}$ tend to zero, (36) becomes

$$
\lambda \approx \mathbf{a}^{T} \cdot \mathbf{S}^{T} \cdot \mathbf{W} \cdot \mathbf{S} \cdot \mathbf{a}+\mathbf{a}^{T} \cdot E\left\{\mathbf{Q}^{T} \cdot \mathbf{W} \cdot \mathbf{Q}\right\} \cdot \mathbf{a}=\mathbf{a}^{T} \cdot \mathbf{\Upsilon} \cdot \mathbf{a} .
$$

Putting (35) into (37) yields

$$
\begin{aligned}
\lambda & \approx \mathbf{a}^{T} \cdot D_{0}\left[\begin{array}{cc}
2\left(1+\cos \left(2 \omega_{1}\right)\right) & 2 \cos \left(\omega_{1}\right) \\
2 \cos \left(\omega_{1}\right) & 1
\end{array}\right] \cdot\left[\begin{array}{c}
1 \\
-2 \cos \left(\omega_{1}\right)
\end{array}\right] \\
& =\mathbf{a}^{T} \cdot D_{0}\left[\begin{array}{l}
0 \\
0
\end{array}\right]=0 .
\end{aligned}
$$

This means that the WLS frequency estimate using the unitnorm constraint is approximately equal to the solution obtained from

$$
\begin{array}{rr}
\mathbf{X}^{T} \cdot \mathbf{W} \cdot \mathbf{X} \cdot \hat{\mathbf{a}}=\mathbf{0} & \text { or } \\
{\left[\begin{array}{ll}
\mathbf{X}_{\mathbf{1}}{ }^{T} \cdot \mathbf{W} \cdot \mathbf{X}_{\mathbf{1}} & \mathbf{X}_{\mathbf{1}}{ }^{T} \cdot \mathbf{W} \cdot \mathbf{X}_{\mathbf{2}} \\
\mathbf{X}_{\mathbf{2}}{ }^{T} \cdot \mathbf{W} \cdot \mathbf{X}_{\mathbf{1}} & \mathbf{X}_{\mathbf{2}}{ }^{T} \cdot \mathbf{W} \cdot \mathbf{X}_{\mathbf{2}}
\end{array}\right]\left[\begin{array}{c}
1 \\
\hat{a}_{1}
\end{array}\right]=\mathbf{0}}
\end{array}
$$

where $\mathbf{0}$ is a zero vector of size 2 , provided that the first element of $\hat{\mathbf{a}}$, namely, $\hat{a}_{0}$, is not zero, and in this sense the constraint of (25) is simply to avoid the trivial solution of all zeros. As one of the eigenvalues of $\mathbf{X}^{T} \cdot \hat{\mathbf{W}} \cdot \mathbf{X}$ approaches zero, the rank of $\mathbf{X}^{T} \cdot \hat{\mathbf{W}} \cdot \mathbf{X}$ will become 1 and hence any one of the two equations in (39) can be used to solve for $\hat{a}_{1}$. In particular, employing the last equation in (39) yields (31), which is the monic-norm solution. As a result, the WLS frequency estimators using the generalized unit-norm and monic constraints will give very close estimates as $N \rightarrow \infty$ and on parameter convergence. In fact, we have demonstrated via computer simulations that for a single real tone, using the upper, lower, or both equations of (39) gives very similar estimation performance for sufficiently large sample lengths. In addition, it can be shown that the two equations will provide equivalent frequency estimates in the absence of noise. It is noteworthy that the nearly equivalence of the two estimators for $L>1$ can also be shown in a similar manner as long as $\lambda \rightarrow 0$.

In the following, we will derive the bias and variance for the monic constraint estimator, and the results should hold for the unit-norm constraint method as $N \rightarrow \infty$. Partitioning $\mathbf{S}$ and $\mathbf{Q}$ into $\left[\begin{array}{ll}\mathbf{S}_{\mathbf{1}} & \mathbf{S}_{\mathbf{2}}\end{array}\right]$ and $\left[\begin{array}{ll}\mathbf{Q}_{\mathbf{1}} & \mathbf{Q}_{\mathbf{2}}\end{array}\right]$ as in (29), and expressing $\mathbf{X}_{\mathbf{2}}$ in terms of $\mathbf{X}_{\mathbf{1}}$ with the use of $\mathbf{S}_{\mathbf{1}}=-a_{1} \cdot \mathbf{S}_{\mathbf{2}}$, (31) becomes

$$
\begin{aligned}
\hat{a}_{1} & =-\frac{\mathbf{X}_{\mathbf{2}}^{T} \cdot \mathbf{W} \cdot\left(-a_{1} \mathbf{X}_{\mathbf{2}}+\mathbf{Q}_{\mathbf{1}}+a_{1} \mathbf{Q}_{\mathbf{2}}\right)}{\mathbf{X}_{\mathbf{2}}^{T} \cdot \mathbf{W} \cdot \mathbf{X}_{\mathbf{2}}} \\
& =a_{1}+\frac{\mathbf{X}_{\mathbf{2}}^{T} \cdot \mathbf{W} \cdot\left(\mathbf{Q}_{\mathbf{1}}+a_{1} \mathbf{Q}_{\mathbf{2}}\right)}{\mathbf{X}_{\mathbf{2}}^{T} \cdot \mathbf{W} \cdot \mathbf{X}_{\mathbf{2}}} .
\end{aligned}
$$

The bias of $\hat{a}_{1}$, denoted by $\Delta \hat{a}_{1}$, is then

$$
\begin{aligned}
& \Delta \hat{a}_{1} \\
& =E\left\{\frac{\mathbf{X}_{\mathbf{2}}^{T} \cdot \mathbf{W} \cdot\left(\mathbf{Q}_{\mathbf{1}}+a_{1} \mathbf{Q}_{\mathbf{2}}\right)}{\mathbf{X}_{\mathbf{2}}^{T} \cdot \mathbf{W} \cdot \mathbf{X}_{\mathbf{2}}}\right\} \approx \frac{E\left\{\mathbf{X}_{\mathbf{2}}^{T} \cdot \mathbf{W} \cdot\left(\mathbf{Q}_{\mathbf{1}}+a_{1} \mathbf{Q}_{\mathbf{2}}\right)\right\}}{E\left\{\mathbf{X}_{\mathbf{2}}^{T} \cdot \mathbf{W} \cdot \mathbf{X}_{\mathbf{2}}\right\}} \\
& \approx \frac{E\left\{\mathbf{X}_{\mathbf{2}}^{T} \cdot \mathbf{U}^{2} \cdot\left(\mathbf{Q}_{\mathbf{1}}+a_{1} \mathbf{Q}_{\mathbf{2}}\right)\right\}}{E\left\{\mathbf{X}_{\mathbf{2}}^{T} \cdot \mathbf{U}^{2} \cdot \mathbf{X}_{\mathbf{2}}\right\}} .
\end{aligned}
$$

Note that the approximation in the expectation is valid because the value of $E\left\{\mathbf{X}_{\mathbf{2}}{ }^{T} \cdot \mathbf{W} \cdot \mathbf{X}_{\mathbf{2}}\right\}$ is dominated by a deterministic term, which is shown in Appendix D. In this Appendix, we have also derived the value of $\Delta \hat{a}_{1}$, which has the form:

$$
\Delta \hat{a}_{1} \approx \frac{-3 \cos \left(\omega_{1}\right)}{\operatorname{SNR}\left((N-2)^{2}-1\right) \sin ^{2}\left(\omega_{1}\right)}
$$

where SNR $=\alpha_{1}^{2} /\left(2 \sigma_{q}^{2}\right)$, which is of order $N^{-2}$. This means that the corresponding bias of the frequency estimate $\hat{\omega}_{1}$ is also $O\left(N^{-2}\right)$ and, thus, is insignificant as $N \rightarrow \infty$.

The variance of $\hat{a}_{1}$ for sufficiently large $N$, denoted by $\operatorname{var}\left(\hat{a}_{1}\right)$, is derived as (see Appendix D)

$$
\operatorname{var}\left(\hat{a}_{1}\right) \approx \frac{48 \sin ^{2}\left(\omega_{1}\right)}{\operatorname{SNR}(N-2)\left((N-2)^{2}-1\right)} .
$$

The relationship between $\operatorname{var}\left(\hat{a}_{1}\right)$ and the variance of $\hat{\omega}_{1}$, denoted by $\operatorname{var}\left(\hat{\omega}_{1}\right)$, is [13]

$$
\operatorname{var}\left(\hat{\omega}_{1}\right) \approx \frac{\operatorname{var}\left(a_{1}\right)}{4 \sin ^{2}\left(\omega_{1}\right)} .
$$

Substituting (43) into (44), we eventually get

$$
\operatorname{var}\left(\hat{\omega}_{1}\right) \approx \frac{12}{\operatorname{SNR}(N-2)\left((N-2)^{2}-1\right)}
$$

which is equal to the CRLB for single-tone frequency estimation in white Gaussian noise [4] as $N \rightarrow \infty$, although our derivation does not necessitate that $q(n)$ is Gaussian. Finally, we should emphasize that the theoretical results are produced under the

$$
\mathbf{U}=\frac{1}{2 \sin \left(\omega_{1}\right)}\left[\begin{array}{ccccc}
0 & \sin \left(\omega_{1}\right) & \sin \left(2 \omega_{1}\right) & \cdots & \sin \left((N-3) \omega_{1}\right) \\
\sin \left(\omega_{1}\right) & 0 & \sin \left(\omega_{1}\right) & \cdots & \sin \left((N-4) \omega_{1}\right) \\
\sin \left(2 \omega_{1}\right) & \sin \left(\omega_{1}\right) & 0 & \cdots & \sin \left((N-5) \omega_{1}\right) \\
\vdots & \vdots & \vdots & \vdots & \vdots \\
\sin \left((N-3) \omega_{1}\right) & \sin \left((N-4) \omega_{1}\right) & \sin \left((N-5) \omega_{1}\right) & \cdots & 0
\end{array}\right] .
$$


assumptions A1) and A2). Nevertheless, the theoretical development provides insightful findings for the performance of the proposed estimators, as are demonstrated in the simulations.

\section{Closed-Form Algorithms for Single-Tone FREQUENCY ESTIMATION}

For single-tone frequency estimation, it is possible to devise closed-form algorithms for the LP-based WLS frequency estimation using the generalized unit-norm and monic constraints. The unit-norm constraint algorithm is first developed as follows. Let $\mathbf{Y}=\mathbf{X}^{T} \cdot \hat{\mathbf{W}} \cdot \mathbf{X}$ which is a $2 \times 2$ matrix for $L=1$. The generalized eigenvalue problem of (26) can now be expressed as

$$
\left[\begin{array}{l}
\hat{a}_{0}[\mathbf{Y}]_{1,1}+\hat{a}_{1}[\mathbf{Y}]_{1,2} \\
\hat{a}_{0}[\mathbf{Y}]_{1,2}+\hat{a}_{1}[\mathbf{Y}]_{2,2}
\end{array}\right]=\lambda\left[\begin{array}{l}
\hat{a}_{0}[\mathbf{\Upsilon}]_{1,1}+\hat{a}_{1}[\mathbf{\Upsilon}]_{1,2} \\
\hat{a}_{0}[\mathbf{\Upsilon}]_{1,2}+\hat{a}_{1}[\mathbf{\Upsilon}]_{2,2}
\end{array}\right]
$$

with the use of $[\mathbf{Y}]_{1,2}=[\mathbf{Y}]_{2,1}$ and $[\mathbf{\Upsilon}]_{1,2}=[\mathbf{\Upsilon}]_{2,1}$. We then eliminate $\lambda$ using (46) and put $\hat{a}_{0}=1$ to yield

$$
\hat{a}_{1}^{2} \beta_{1}+\hat{a}_{1} \beta_{2}+\beta_{3}=0
$$

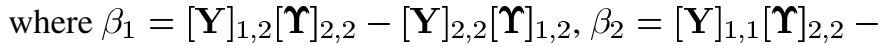

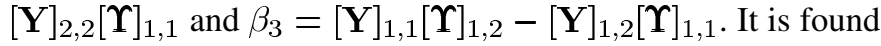
that the root which corresponds to the frequency estimate is

$$
\hat{a}_{1}=-\frac{\beta_{2}+\sqrt{\beta_{2}^{2}-4 \beta_{1} \beta_{3}}}{2 \beta_{2}} .
$$

As a result, the closed-form algorithm using the unit-norm constraint for single-tone frequency estimation is summarized as follows.

i) Find an initial estimate of $a_{1}$ using (16) or other methods such as finding the discrete Fourier transform (DFT) peak of $x(n)$.

ii) Use the estimated $\hat{a}_{1}$ to obtain $\mathbf{Y}$ and $\boldsymbol{\Upsilon}$.

iii) Compute an improved estimate of $a_{1}$ using (48).

iv) Repeat Steps ii) and iii) until parameter convergence.

v) Compute the frequency estimate using $\cos ^{-1}\left(-\hat{a}_{1} / 2\right)$.

For the closed-form algorithm using the monic constraint, we notice that $\mathbf{X}_{\mathbf{2}}{ }^{T} \cdot \hat{\mathbf{W}} \cdot \mathbf{X}_{\mathbf{2}}$ and $\mathbf{X}_{\mathbf{2}}{ }^{T} \cdot \hat{\mathbf{W}} \cdot \mathbf{X}_{\mathbf{1}}$ of (31) are scalars when $L=1$. Similarly, the corresponding iterative estimation procedure is as follows.

i) Find an initial estimate of $a_{1}$ using (16) or otherwise.

ii) Use the estimated $\hat{a}_{1}$ to obtain $\hat{\mathbf{W}}$.

iii) Compute an improved estimate of $a_{1}$ using $-\mathbf{X}_{\mathbf{2}}{ }^{T} \cdot \hat{\mathbf{W}}$. $\mathbf{X}_{1} /\left(\mathbf{X}_{\mathbf{2}}^{T} \cdot \hat{\mathbf{W}} \cdot \mathbf{X}_{\mathbf{2}}\right)$.

iv) Repeat Steps ii) and iii) until parameter convergence.

v) Compute the frequency estimate using $\cos ^{-1}\left(-\hat{a}_{1} / 2\right)$.

\section{Simulation Results}

Computer simulations had been carried out to evaluate the frequency estimation performance of the proposed algorithms in white Gaussian noise. Comparisons were also made with the CRLB [4]. Unless stated otherwise, the two algorithms were initiated by the RPHD. All simulation results provided were averages of 1000 independent runs.

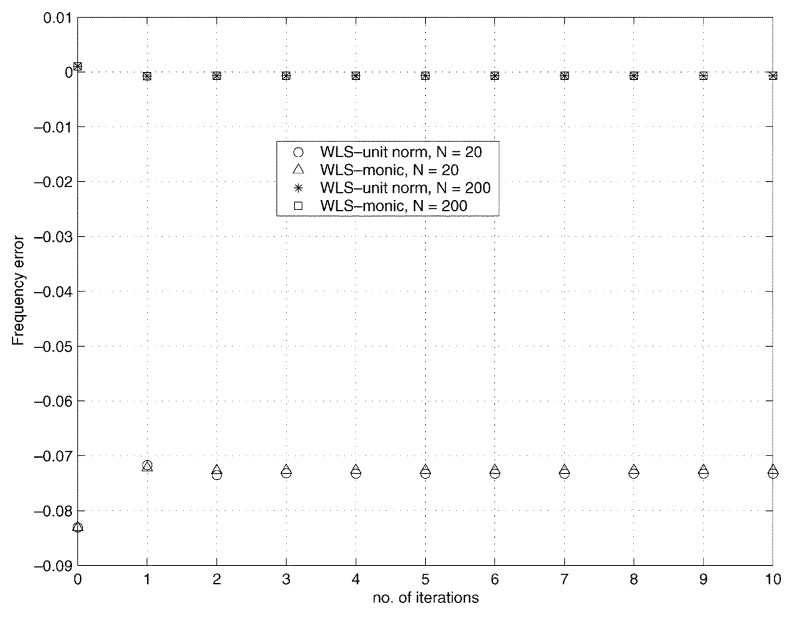

Fig. 1. Frequency errors versus number of iterations at $\omega_{1}=0.3 \pi$ and $\mathrm{SNR}=10 \mathrm{~dB}$ for $N=20$ and $N=200$.

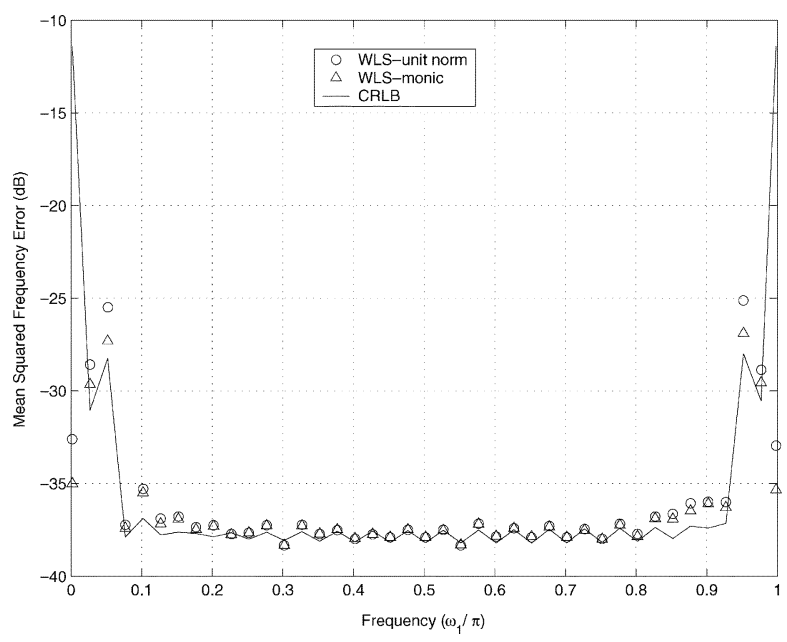

Fig. 2. Mean squared frequency errors versus $\omega_{1}$ at $\mathrm{SNR}=10 \mathrm{~dB}$ and $N=$ 20 with one iteration.

Fig. 1 illustrates typical realizations of the frequency errors in a single run versus the number of iterations in the proposed algorithms for a single real sinusoid with $\alpha_{1}=\sqrt{2}, \omega_{1}=0.3 \pi$ and $\phi_{1}=0$ with $\sigma_{q}^{2}=0.1$, which corresponded to a SNR of $10 \mathrm{~dB}$. The frequency errors were obtained by subtracting $\omega_{1}$ from the corresponding frequency estimates and the scenarios of $N=20$ and $N=200$ were considered. The iteration number of 0 referred to the frequency errors based on the initial estimates of (16). Obvious changes in frequency errors are observed from the zeroth to first iterations. Furthermore, it is seen that at most three iterations were required to attain a nearly converged estimate, which implies that noticeable improvement in performance will not be acquired if more iterations are employed. The cases of using one and three iterations, which corresponded to generally nonconverged and almost converged estimates, respectively, were investigated as follows.

Fig. 2 shows the mean squared frequency errors (MSFEs) of the two methods as well the CRLB for a single real sinusoid with $\alpha_{1}=\sqrt{2}, \phi_{1}=0$ and $N=20$ at SNR $=10 \mathrm{~dB}$. Step (iv) in the algorithms was skipped so that only one iteration was employed. We observe that the MSFEs of the proposed methods had similar values and were close to the CRLB for $\omega_{1} \in[0.2,0.8] \pi$. 


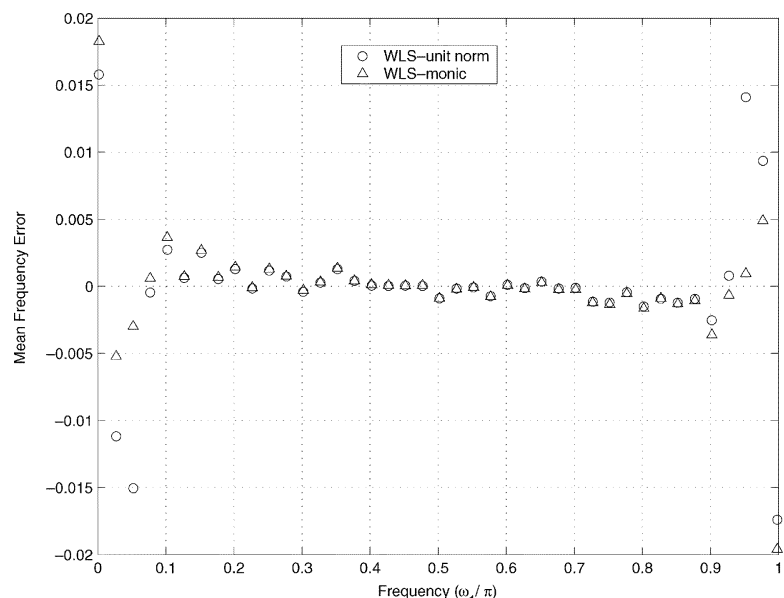

Fig. 3. Mean frequency errors versus $\omega_{1}$ at $\mathrm{SNR}=10 \mathrm{~dB}$ and $N=20$ with one iteration.

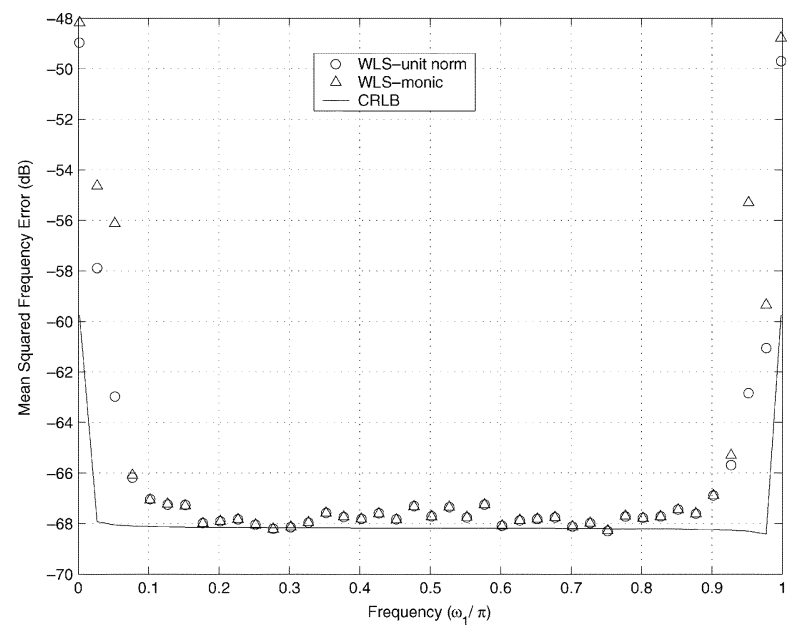

Fig. 4. Mean squared frequency errors versus $\omega_{1}$ at $\mathrm{SNR}=10 \mathrm{~dB}$ and $N=$ 200 with one iteration.

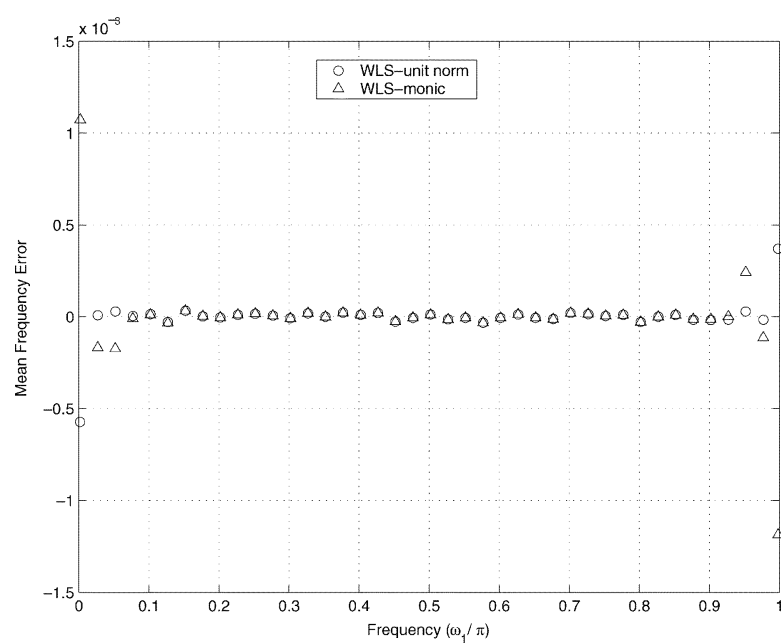

Fig. 5. Mean frequency errors versus $\omega_{1}$ at $\mathrm{SNR}=10 \mathrm{~dB}$ and $N=200$ with one iteration.

The mean frequency errors are shown in Fig. 3 and it is seen that the biases in the WLS schemes were small except when $\omega_{1}$ was near 0 or $\pi$. This test was repeated for $N=200$ and

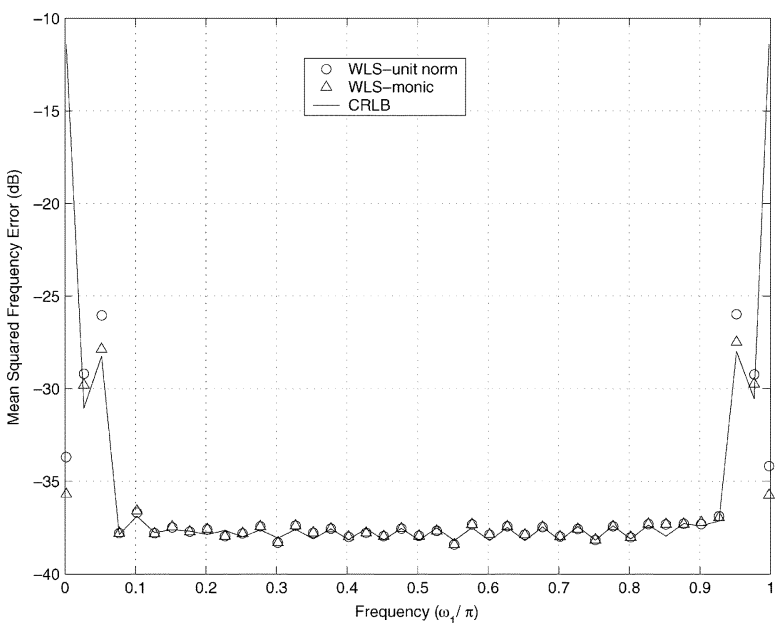

Fig. 6. Mean squared frequency errors versus $\omega_{1}$ at $\mathrm{SNR}=10 \mathrm{~dB}$ and $N=$ 20 with three iterations.

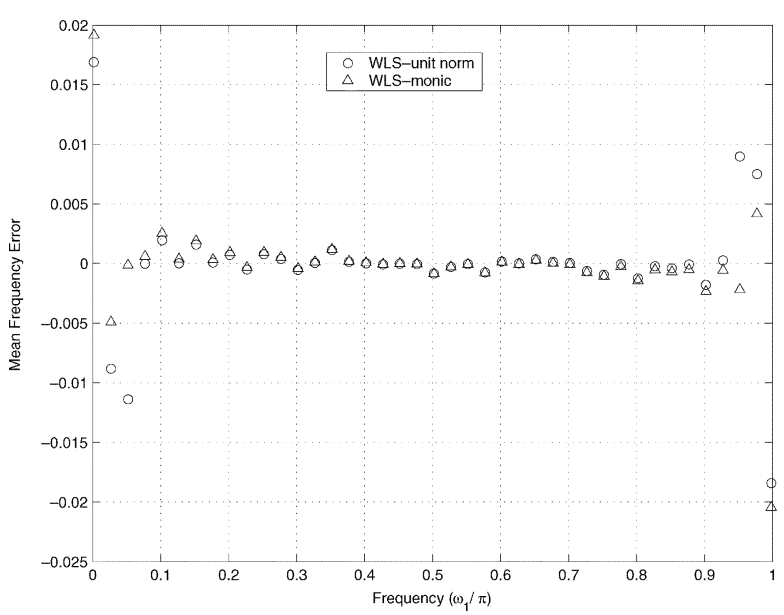

Fig. 7. Mean frequency errors versus $\omega_{1}$ at $\mathrm{SNR}=10 \mathrm{~dB}$ and $N=20$ with three iterations.

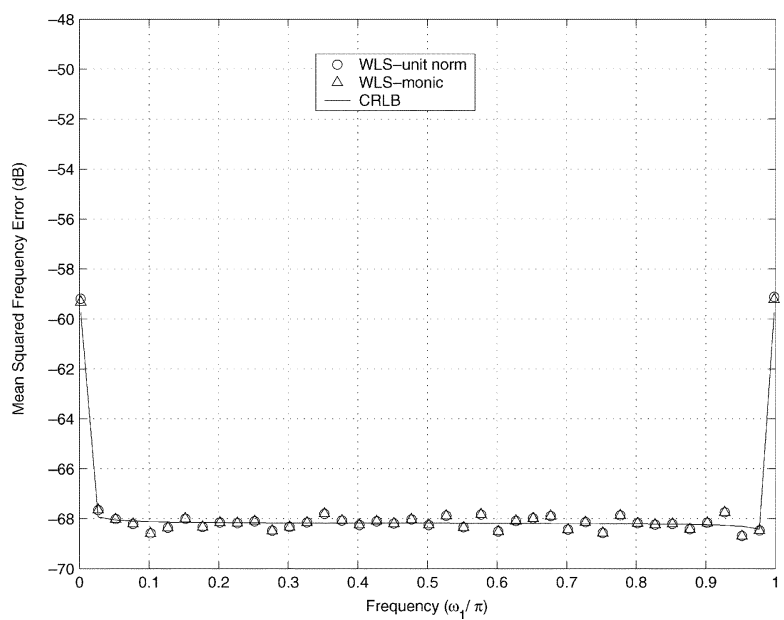

Fig. 8. Mean squared frequency errors versus $\omega_{1}$ at $\mathrm{SNR}=10 \mathrm{~dB}$ and $N=$ 200 with three iterations.

the results are plotted in Figs. 4 and 5. It is observed that both estimators had very similar MSFEs and mean frequency errors for $\omega_{1} \in[0.1,0.9] \pi$. Moreover, their estimation performance was comparable with the CRLB when $\omega_{1}$ was neither near 0 


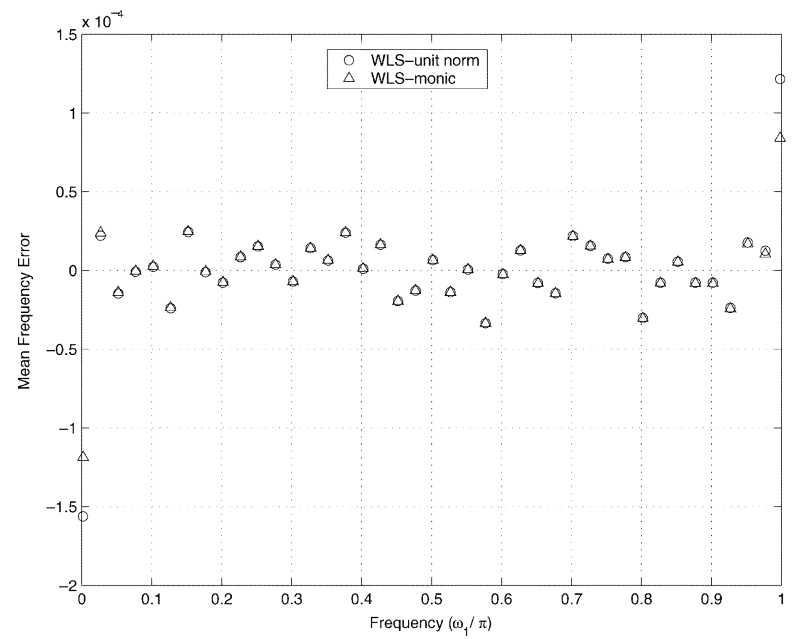

Fig. 9. Mean frequency errors versus $\omega_{1}$ at $\mathrm{SNR}=10 \mathrm{~dB}$ and $N=200$ with three iterations.

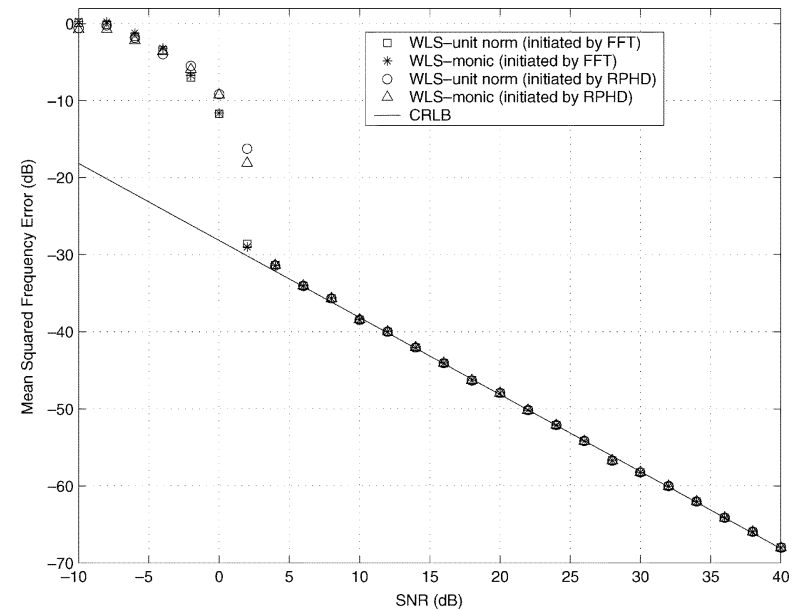

Fig. 10. Mean squared frequency errors versus SNR at $\omega_{1}=0.3 \pi$ and $N=$ 20 with three iterations.



Fig. 11. Mean squared frequency errors versus SNR at $\omega_{1}=0.3 \pi$ and $N=$ 1000 with three iterations.

nor $\pi$. The above experiments were repeated using three iterations in the estimation procedures and the results are shown in Figs. 6-9. It is observed that the two algorithms provided very



Fig. 12. Mean squared frequency errors versus $N$ at $\omega_{1}=0.3 \pi$ and $\mathrm{SNR}=$ $0 \mathrm{~dB}$ with three iterations.

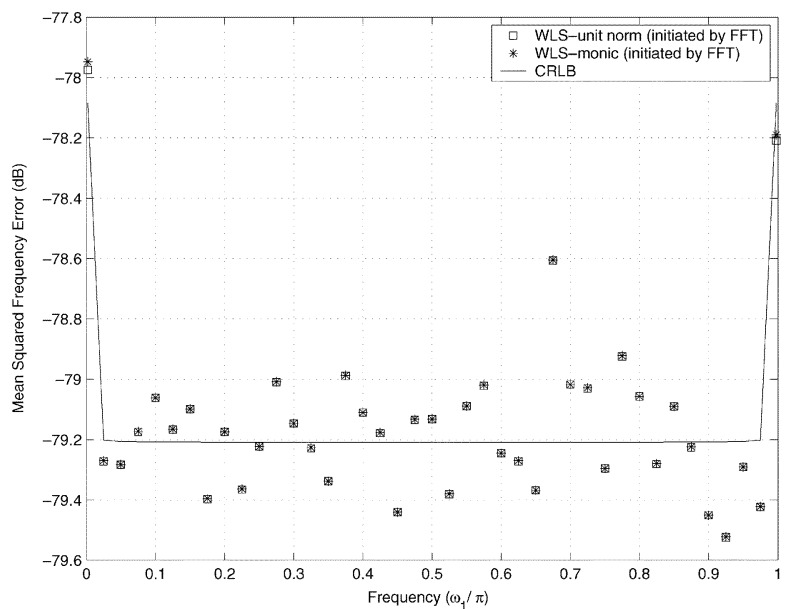

Fig. 13. Mean squared frequency errors versus $\omega_{1}$ at $\mathrm{SNR}=0 \mathrm{~dB}$ and $N=$ 1000 with three iterations.

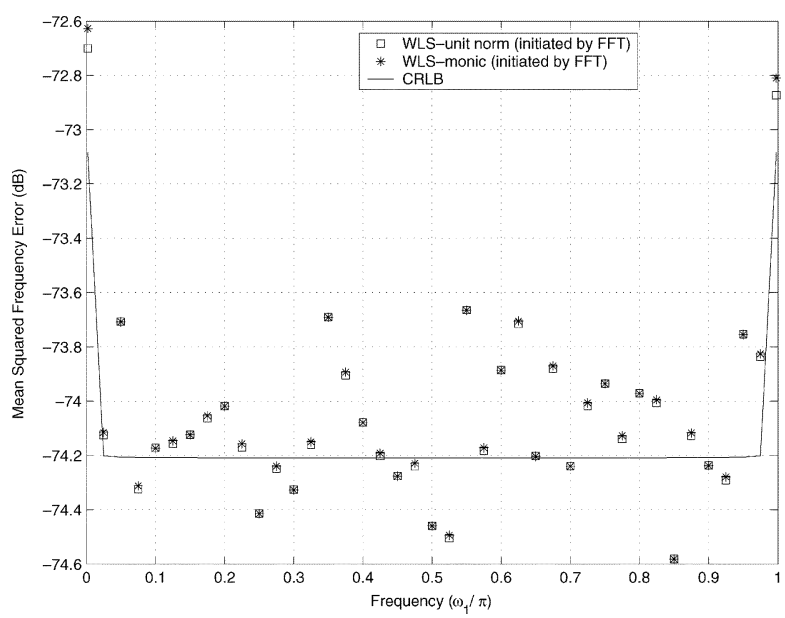

Fig. 14. Mean squared frequency errors versus $\omega_{1}$ at $\mathrm{SNR}=-5 \mathrm{~dB}$ and $N=1000$ with three iterations.

similar estimation accuracies and approached the CRLB. These results indicated the optimality of the two methods upon parameter convergence even for a short data length. 


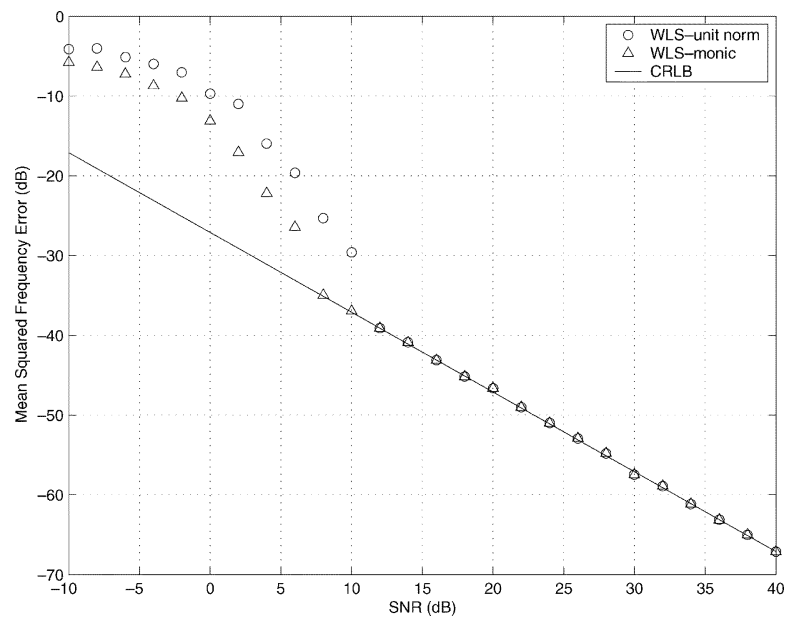

Fig. 15. Mean squared frequency errors for $\omega_{1}$ versus SNR at $\omega_{1}=0.3 \pi$, $\omega_{2}=0.7 \pi$ and $N=20$ with three iterations.

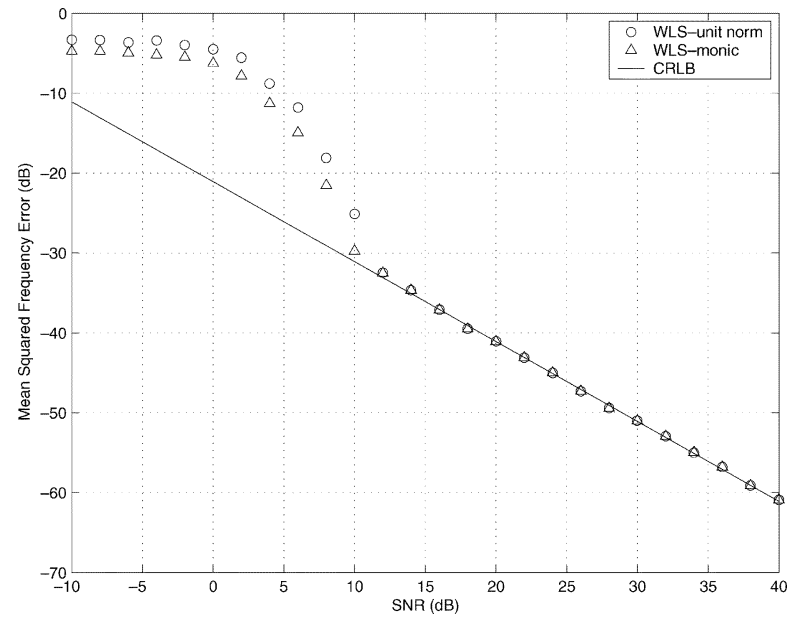

Fig. 16. Mean squared frequency errors for $\omega_{2}$ versus SNR at $\omega_{1}=0.3 \pi$, $\omega_{2}=0.7 \pi$ and $N=20$ with three iterations.

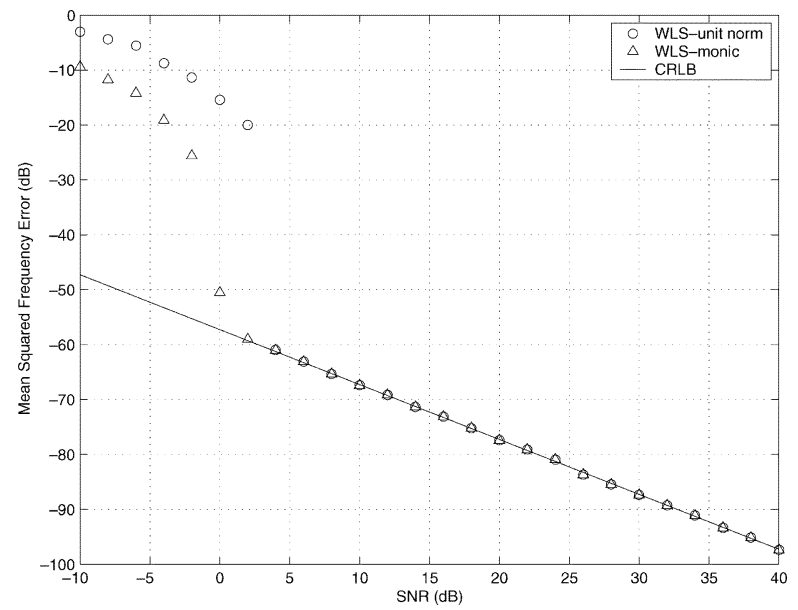

Fig. 17. Mean squared frequency errors for $\omega_{1}$ versus SNR at $\omega_{1}=0.3 \pi$, $\omega_{2}=0.7 \pi$ and $N=200$ with three iterations.

Fig. 10 plots the MSFEs of the WLS algorithms versus SNR at $N=20$ and $\omega_{1}=0.3 \pi$ with three iterations. Initialization using the DFT peak was also included. We see that both algorithms with different initialization methods had the similar

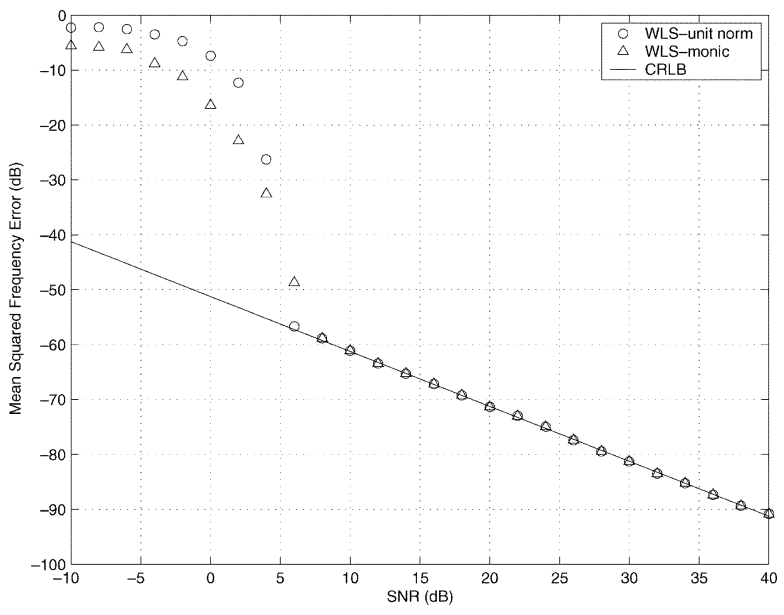

Fig. 18. Mean squared frequency errors for $\omega_{2}$ versus SNR at $\omega_{1}=0.3 \pi$, $\omega_{2}=0.7 \pi$ and $N=200$ with three iterations.

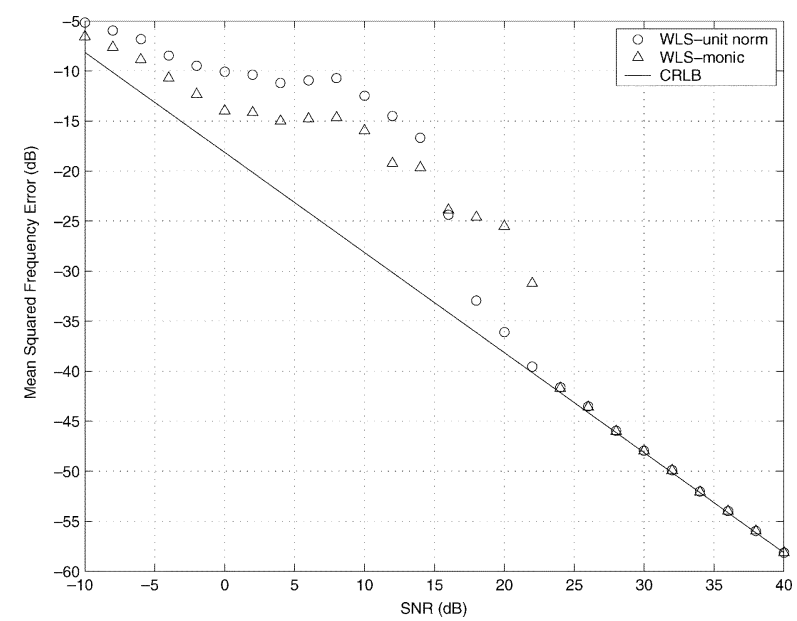

Fig. 19. Mean squared frequency errors for $\omega_{1}$ versus SNR at $\omega_{1}=0.3 \pi$, $\omega_{2}=0.34 \pi$ and $N=20$ with three iterations.

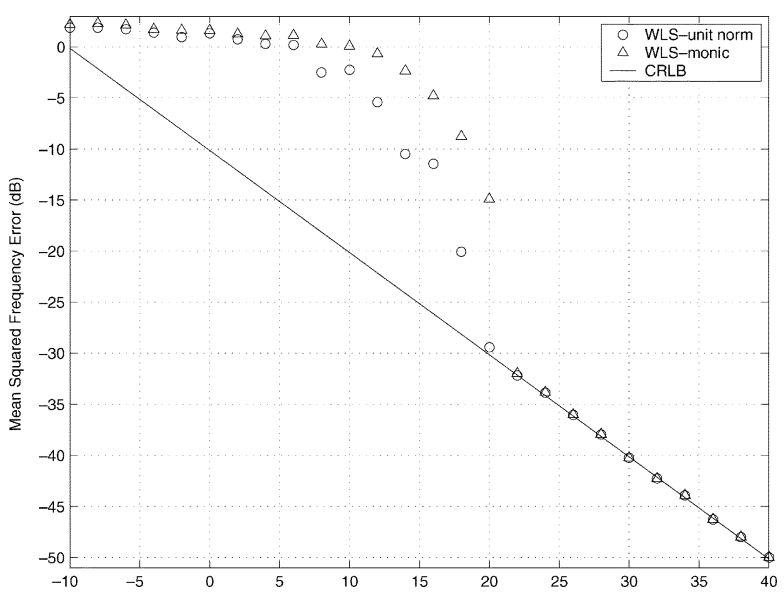

Fig. 20. Mean squared frequency errors for $\omega_{2}$ versus SNR at $\omega_{1}=0.3 \pi$, $\omega_{2}=0.34 \pi$ and $N=20$ with three iterations.

threshold SNRs, with deviation of around $2 \mathrm{~dB}$, and attained the CRLB for sufficiently high SNR conditions. This test was repeated for $N=1000$ and the results are shown in Fig. 11. It is observed that their threshold SNRs decreased, especially for 


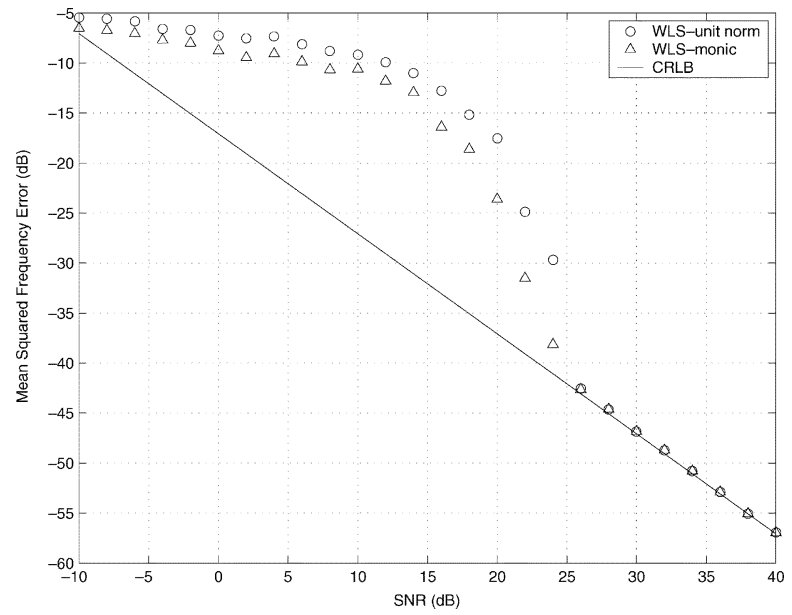

Fig. 21. Mean squared frequency errors for $\omega_{1}$ versus SNR at $\omega_{1}=0.3 \pi$, $\omega_{2}=0.34 \pi, \omega_{2}=0.7 \pi$ and $N=20$ with three iterations.

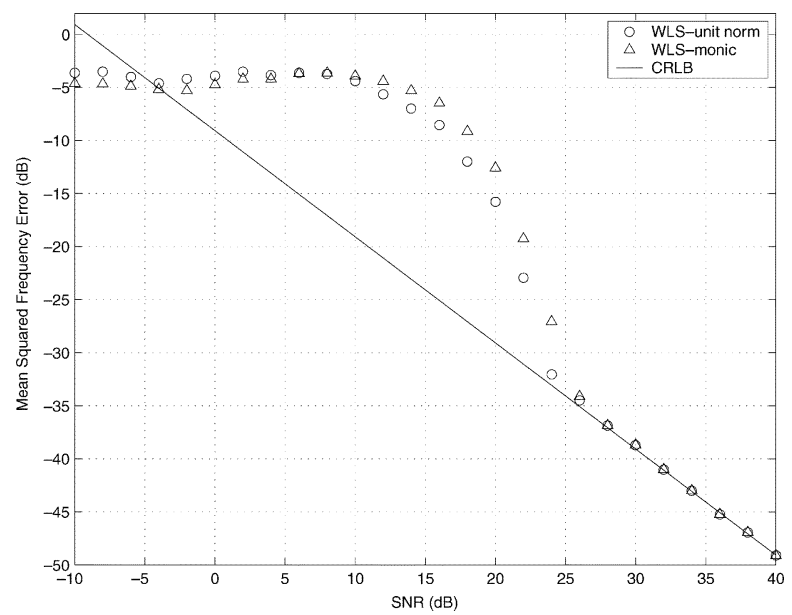

Fig. 22. Mean squared frequency errors for $\omega_{2}$ versus SNR at $\omega_{1}=0.3 \pi$, $\omega_{2}=0.34 \pi, \omega_{2}=0.7 \pi$ and $N=20$ with three iterations.

the DFT initialization, which corresponded a larger SNR operation range, as $N$ increased. Fig. 12 plots the MSFEs versus $N$ at $\omega_{1}=0.3 \pi$ and SNR $=0 \mathrm{~dB}$. The plots of MSFEs versus frequency at $N=1000$ for $\mathrm{SNR}=0 \mathrm{~dB}$ and $\mathrm{SNR}=-5 \mathrm{~dB}$ are shown Figs. 13 and 14, respectively, where DFT was used as initialization while all other simulation settings were same as those in Fig. 2. It is observed that accurate frequency estimation was achieved in both small SNR cases. From Figs. 10-14, we summarize that the two proposed estimators can produce optimum estimation performance when the data lengths and/or SNRs are sufficiently large. Furthermore, for single-tone frequency estimation under lower SNR conditions, initialization with DFT will give more robust estimates than using RPHD.

Figs. 15 and 16 show the MSFEs of the methods versus SNR for the case of two sinusoids at $N=20$ with three iterations. The parameters of the sinusoids were given as follows: $\alpha_{1}=$ $\sqrt{2}, \alpha_{2}=\sqrt{2} / 2, \omega_{1}=0.3 \pi, \omega_{2}=0.7 \pi$ and $\phi_{1}=\phi_{2}=0$. It is observed that the two algorithms were optimum when the SNR was sufficiently high, with the monic constraint method has relatively smaller threshold SNRs for both frequencies. The test for dual tones was repeated for $N=200$ and the results are shown in Figs. 17 and 18. The optimality of the two algorithms

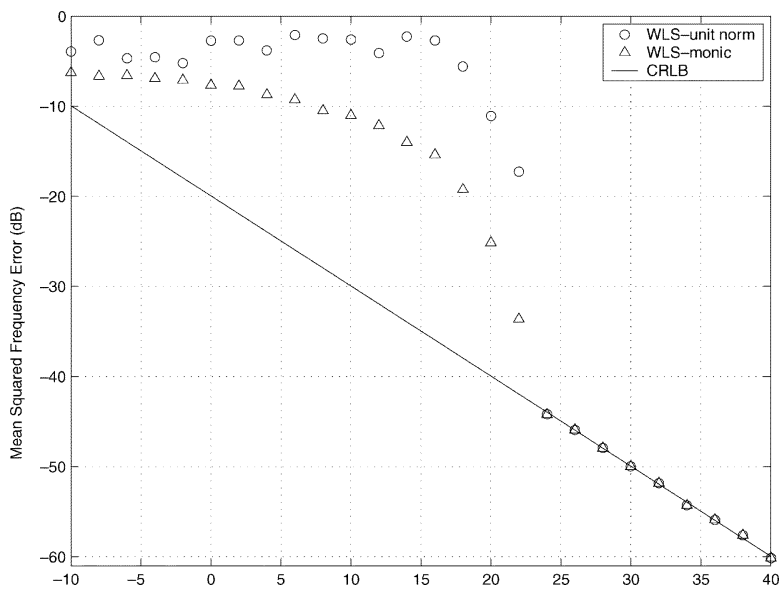

Fig. 23. Mean squared frequency errors for $\omega_{3}$ versus SNR at $\omega_{1}=0.3 \pi$, $\omega_{2}=0.34 \pi, \omega_{2}=0.7 \pi$ and $N=20$ with three iterations.

was again demonstrated for sufficiently high SNRs. Finally, the high-resolution frequency estimation property of the algorithms is demonstrated in Figs. 19-23, for $L=2$ and $L=3$. The parameters in the two-tone and three-tone cases were given as follows, $\alpha_{1}=\sqrt{2}, \alpha_{2}=\sqrt{2} / 2, \omega_{1}=0.3 \pi, \omega_{2}=0.34 \pi$ and $\phi_{1}=\phi_{2}=0$, and $\alpha_{1}=\sqrt{2}, \alpha_{2}=\sqrt{2} / 2, \alpha_{3}=\sqrt{2} / 2$, $\omega_{1}=0.3 \pi, \omega_{2}=0.34 \pi, \omega_{2}=0.7 \pi$ and $\phi_{1}=\phi_{2}=\phi_{3}=0$.

\section{CONCLUSIONS}

Two accurate frequency estimation algorithms for multiple real sinusoids in white noise based on the linear prediction approach have been developed. The first algorithm minimizes a weighted least squares (WLS) cost function subject to a generalized unit-norm constraint while the second method is a WLS estimator with monic constraint. For a single real tone, both algorithms give very close frequency estimates whose accuracies attain Cramér-Rao lower bound for white Gaussian noise. Closed-form single-tone frequency estimation is also investigated. Moreover, the optimality of the proposed algorithms for single and multiple frequency estimation is demonstrated via computer simulations at different frequencies, data lengths, and signal-to-noise ratio conditions.

\section{APPENDIX A}

The derivation of (23) is given as follows. We first decompose $\mathrm{Q}$ as

$$
\begin{aligned}
& \mathbf{Q}=\mathbf{G}+\mathbf{H} \\
& \text { where } \\
& \mathbf{G}=\left[\begin{array}{cccc}
q(N-1) & \cdots & q(N-L) & q(N-L-1) \\
q(N-2) & \cdots & q(N-L-1) & q(N-L-2) \\
\vdots & \vdots & \vdots & \vdots \\
q(2 L) & \cdots & q(L+1) & q(L)
\end{array}\right] \\
& \text { and } \\
& \mathbf{H}=\left[\begin{array}{cccc}
q(N-2 L-1) & \cdots & q(N-L-2) & 0 \\
q(N-2 L-2) & \cdots & q(N-L-3) & 0 \\
\vdots & \vdots & \vdots & \vdots \\
q(0) & \cdots & q(L-1) & 0
\end{array}\right] .
\end{aligned}
$$


The expected value of $E\left\{\mathbf{Q}^{T} \cdot \mathbf{W} \cdot \mathbf{Q}\right\}$ is then expanded as

$$
\begin{array}{r}
E\left\{\mathbf{Q}^{T} \cdot \mathbf{W} \cdot \mathbf{Q}\right\}=E\left\{\mathbf{G}^{T} \cdot \mathbf{W} \cdot \mathbf{G}\right\}+E\left\{\mathbf{G}^{T} \cdot \mathbf{W} \cdot \mathbf{H}\right\} \\
+E\left\{\mathbf{H}^{T} \cdot \mathbf{W} \cdot \mathbf{G}\right\}+E\left\{\mathbf{H}^{T} \cdot \mathbf{W} \cdot \mathbf{H}\right\} .
\end{array}
$$

Since $q(n)$ is a white process with variance $\sigma_{q}^{2}$, the value of the $(m, n)$ th element of $E\left\{\mathbf{G}^{T} \cdot \mathbf{W} \cdot \mathbf{G}\right\}$ is observed as

$$
\begin{aligned}
& \sum_{i=1}^{N-2 L} \sum_{j=1}^{N-2 L} E\left\{[\mathbf{G}]_{i, m}[\mathbf{W}]_{i, j}[\mathbf{G}]_{j, n}\right\} \\
& =\sum_{i=1}^{N-2 L-|m-n|} E\left\{[\mathbf{G}]_{i, m}[\mathbf{W}]_{i, i+|m-n|}[\mathbf{G}]_{i+|m-n|, n}\right\} .
\end{aligned}
$$

We then deduce from (A.2) that

$$
E\left\{\mathbf{G}^{T} \cdot \mathbf{W} \cdot \mathbf{G}\right\}=\left[\begin{array}{cccc}
D_{0} & D_{1} & \cdots & D_{L} \\
D_{1} & D_{0} & \cdots & D_{L-1} \\
\vdots & \vdots & \vdots & \vdots \\
D_{L} & D_{L-1} & \cdots & D_{0}
\end{array}\right] \sigma_{q}^{2}
$$

In a similar manner, it is shown that

$E\left\{\mathbf{G}^{T} \cdot \mathbf{W} \cdot \mathbf{H}\right\}=\left[\begin{array}{ccccc}D_{2 L} & D_{2 L-1} & \cdots & D_{L-1} & 0 \\ D_{2 L-1} & D_{2 L-2} & \cdots & D_{L-2} & 0 \\ \vdots & \vdots & \vdots & \vdots & \vdots \\ D_{L} & D_{L-1} & \cdots & D_{1} & 0\end{array}\right] \sigma_{q}^{2}$

and

$$
E\left\{\mathbf{H}^{T} \cdot \mathbf{W} \cdot \mathbf{H}\right\}=\left[\begin{array}{ccccc}
D_{0} & D_{1} & \cdots & D_{L-1} & 0 \\
D_{1} & D_{0} & \cdots & D_{L-2} & 0 \\
\vdots & \vdots & \vdots & \vdots & \vdots \\
D_{L-1} & D_{L-2} & \cdots & D_{0} & 0 \\
0 & 0 & \cdots & 0 & 0
\end{array}\right] \sigma_{q}^{2} .
$$

Substituting (A.3)-(A.5) into (A.1) yields (23).

\section{APPENDIX B}

The development of the approximate form for $\mathbf{W}$ is inspired by the fact that i) $\mathbf{W}^{-1}$ can be approximated as the square of a tridiagonal matrix; ii) a special tri-diagonal matrix [30], [31] has a closed-form inverse; and iii) the structure of the square of the special tri-diagonal matrix is similar to that of $\mathbf{W}^{-1}$. We first notice from (33) that $\mathbf{W}^{-1} / \sigma_{q}^{2}$ can be approximated by

$\frac{\mathbf{W}^{-1}}{\sigma_{q}^{2}} \approx\left[\begin{array}{ccccccc}1+a_{1}^{2} & 2 a_{1} & 1 & 0 & \cdots & 0 & 0 \\ 2 a_{1} & 2+a_{1}^{2} & 2 a_{1} & 1 & \cdots & 0 & 0 \\ \vdots & \vdots & \vdots & \vdots & \vdots & \vdots & \vdots \\ 0 & 0 & 0 & 0 & \cdots & 2+a_{1}^{2} & 2 a_{1} \\ 0 & 0 & 0 & 0 & \cdots & 2 a_{1} & 1+a_{1}^{2}\end{array}\right]=\mathbf{A}^{2}$ where

$$
\mathbf{A}=\left[\begin{array}{ccccccc}
a_{1} & 1 & 0 & 0 & 0 & \cdots & 0 \\
1 & a_{1} & 1 & 0 & 0 & \cdots & 0 \\
0 & 1 & a_{1} & 1 & 0 & \cdots & 0 \\
\vdots & \vdots & \vdots & \vdots & \vdots & \vdots & \vdots \\
0 & 0 & 0 & 0 & 0 & \cdots & a_{1}
\end{array}\right]
$$

That is, $\mathbf{W}^{-1} / \sigma_{q}^{2}$ is identical to $\mathbf{A}^{2}$, except for the first and last elements.

From [30] and [31], the inverse of the correlation matrix $\mathbf{R}$, which is expressed as

$$
\mathbf{R}=\frac{r}{r^{2}-1}\left[\begin{array}{ccccc}
1 & r & r^{2} & \cdots & r^{N-3} \\
r & 1 & r & \cdots & r^{N-4} \\
\vdots & \vdots & \vdots & \vdots & \vdots \\
r^{N-3} & r^{N-4} & r^{N-5} & \cdots & 1
\end{array}\right]
$$

is given by

$$
\mathbf{T}=\left[\begin{array}{ccccccc}
-\frac{1}{r} & 1 & 0 & 0 & \cdots & 0 & 0 \\
1 & -\frac{1+r^{2}}{r} & 1 & 0 & \cdots & 0 & 0 \\
0 & 1 & -\frac{1+r^{2}}{r} & 1 & \cdots & 0 & 0 \\
\vdots & \vdots & \vdots & \vdots & \vdots & \vdots & \vdots \\
0 & 0 & 0 & 0 & \cdots & -\frac{1+r^{2}}{r} & 1 \\
0 & 0 & 0 & 0 & \cdots & 1 & -\frac{1}{r}
\end{array}\right] .
$$

Ignoring the first and last elements of $\mathbf{A}$, we have $\mathbf{A} \approx \mathbf{T}$ via the establishment of the following relationship:

$$
a_{1}=-2 \cos \left(\omega_{1}\right)=-\frac{1+r^{2}}{r} \Rightarrow r=\exp \left( \pm j \omega_{1}\right)
$$

An obvious closed-form expression for $\mathbf{W}$ at $N \rightarrow \infty$ is then $\mathbf{W} \sigma_{q}^{2} \approx \mathbf{R}^{2}$ with $r=\exp \left(j \omega_{1}\right)$ or $r=\exp \left(-j \omega_{1}\right)$, but it is inappropriate because the corresponding elements of $\mathbf{R}^{2}$ are generally complex, whereas $\mathbf{W}$ should be real. To find a real approximation matrix, we notice from (B.5) that $\mathbf{A}$ is also approximately equal to $\mathbf{T}^{*}$ for $r=\exp \left(j \omega_{1}\right)$ (or $r=\exp \left(-j \omega_{1}\right)$ ), where * represents the conjugate operation. This means that $\mathbf{A}^{-1}$ is approximately equal to $\mathbf{T}^{-1}$ as well as $\left(\mathbf{T}^{*}\right)^{-1}$ for this particular choice of $r$. Therefore, a real approximation for $\mathbf{A}^{-1}$, namely, $\mathbf{U}$, can be found between $\mathbf{T}^{-1}$ and $\left(\mathbf{T}^{*}\right)^{-1}$, and a good choice is simply their standard mean, as in (B.6), shown at the bottom of the next page, where $\Re$ denotes the real part. As a result, we have

$$
\mathbf{W} \sigma_{q}^{2} \approx \mathbf{U}^{2}
$$

By applying the matrix inversion by partitioning lemma [32] and the using mathematical induction, we have proved that the inverse of $\mathbf{U}$ is in (B.8), shown at the bottom of the next page. 
Using (B.8), it is shown that $\mathbf{U}^{-2}=\mathbf{W}^{-1} / \sigma_{q}^{2}$, except for 12 elements at the four corners:

$$
\begin{aligned}
& {\left[\mathbf{U}^{-2}\right]_{1,1} }=\left[\mathbf{U}^{-2}\right]_{N-2, N-2} \\
&=\frac{\sin ^{2}\left(\omega_{1}\right)+\sin ^{2}\left((N-4) \omega_{1}\right)+\sin ^{2}\left((N-3) \omega_{1}\right)}{\sin ^{2}\left((N-3) \omega_{1}\right)} \\
& {\left[\mathbf{U}^{-2}\right]_{1,2} }=\left[\mathbf{U}^{-2}\right]_{2,1}=\left[\mathbf{U}^{-2}\right]_{N-2, N-3}=\left[\mathbf{U}^{-2}\right]_{N-3, N-2} \\
&=-\frac{\sin \left((N-2) \omega_{1}\right)+2 \sin \left((N-4) \omega_{1}\right)}{\sin \left((N-3) \omega_{1}\right)} \\
& {\left[\mathbf{U}^{-2}\right]_{1, N-3} }=\left[\mathbf{U}^{-2}\right]_{N-3,1}=\left[\mathbf{U}^{-2}\right]_{2, N-2} \\
&=\left[\mathbf{U}^{-2}\right]_{N-2,2}=\frac{\sin \left(\omega_{1}\right)}{\sin \left((N-3) \omega_{1}\right)} \\
& \text { and }
\end{aligned}
$$

$$
\left[\mathbf{U}^{-2}\right]_{1, N-2}=\left[\mathbf{U}^{-2}\right]_{N-2,1}=-\frac{2 \sin \left(\omega_{1}\right) \sin \left((N-4) \omega_{1}\right)}{\sin ^{2}\left((N-3) \omega_{1}\right)} .
$$

Using the matrix inversion lemma [23], it can be shown that (B.7) holds very well except for the elements near the matrix boundaries. The result for (B.7) has also been extended for $L>$ 1 as

$$
\mathbf{W} \sigma_{q}^{2} \approx \prod_{l=1}^{L} \mathbf{U}_{l}^{2}
$$

where each $\mathbf{U}_{l}$ is parameterized by the frequency $\omega_{l}$, $l=1,2, \ldots, L$, as in (B.7), and $\prod_{l=1}^{L} \mathbf{U}_{L+1-l}^{-2}$ differs from $\mathbf{W}^{-1} / \sigma_{q}^{2}$ for the $4 L(2 L+1)$ elements at the four corners.

\section{APPENDIX C}

The values for $D_{0}, D_{1}$ and $D_{2}$ in (32) are first derived for $N \rightarrow \infty$ as follows. Using (B.6), $D_{0}$ is computed as

$$
\begin{aligned}
D_{0}= & \sum_{i=1}^{N-2}[\mathbf{W}]_{i, i} \\
\approx & \sigma_{q}^{-2} \operatorname{tr}\left(\mathbf{U}^{2}\right)=\sigma_{q}^{-2} \sum_{i=1}^{N-2} \sum_{j=1}^{N-2}[\mathbf{U}]_{i, j}[\mathbf{U}]_{i, j} \\
= & \frac{1}{\sigma_{q}^{2}} \sum_{k=1}^{N-2}\left(\sum_{i=1}^{N-k-2} \sin ^{2}\left(i \omega_{1}\right)+\sum_{i=1}^{k-1} \sin ^{2}\left(i \omega_{1}\right)\right) \\
& \times\left(\frac{1}{4 \sin ^{2}\left(\omega_{1}\right)}\right) \\
= & \frac{1}{2 \sigma_{q}^{2}} \sum_{k=1}^{N-2}\left(\sum_{i=1}^{N-k-2}\left(1-\cos \left(2 i \omega_{1}\right)\right)+\sum_{i=1}^{k-1}\left(1-\cos \left(2 i \omega_{1}\right)\right)\right. \\
& \times\left(\frac{1}{4 \sin ^{2}\left(\omega_{1}\right)}\right) \\
= & \frac{1}{2 \sigma_{q}^{2}}\left(\frac{(N-2)(N-3)}{N-2}\left(\sum_{i=1}^{N-k-2} \cos \left(2 i \omega_{1}\right)+\sum_{i=1}^{k-1} \cos \left(2 i \omega_{1}\right)\right)\right) \\
& -\sum_{k=1}^{N}\left(\frac{1}{4 \sin ^{2}\left(\omega_{1}\right)}\right) \\
& \times\left(\sum_{i=1}\left(\sum_{i}\right)\right.
\end{aligned}
$$

$$
\begin{aligned}
& \mathbf{U}=\left.\frac{\mathbf{T}^{-1}+\left(\mathbf{T}^{*}\right)^{-1}}{2}\right|_{r=\exp \left(j \omega_{1}\right)} \\
& =\Re\left\{\left.\frac{r}{r^{2}-1}\left[\begin{array}{ccccc}
1 & r & r^{2} & \cdots & r^{N-3} \\
r & 1 & r & \cdots & r^{N-4} \\
\vdots & \vdots & \vdots & \vdots & \vdots \\
r^{N-3} & r^{N-4} & r^{N-5} & \cdots & 1
\end{array}\right]\right|_{r=\exp \left(j \omega_{1}\right)}\right\} \\
& =\frac{1}{2 \sin \left(\omega_{1}\right)}\left[\begin{array}{ccccc}
0 & \sin \left(\omega_{1}\right) & \sin \left(2 \omega_{1}\right) & \cdots & \sin \left((N-3) \omega_{1}\right) \\
\sin \left(\omega_{1}\right) & 0 & \sin \left(\omega_{1}\right) & \cdots & \sin \left((N-4) \omega_{1}\right) \\
\sin \left(2 \omega_{1}\right) & \sin \left(\omega_{1}\right) & 0 & \cdots & \sin \left((N-5) \omega_{1}\right) \\
\vdots & \vdots & \vdots & \vdots & \vdots \\
\sin \left((N-3) \omega_{1}\right) & \sin \left((N-4) \omega_{1}\right) & \sin \left((N-5) \omega_{1}\right) & \cdots & 0
\end{array}\right]
\end{aligned}
$$

$$
\mathbf{U}^{-1}=\left[\begin{array}{ccccccc}
-\frac{\sin \left((N-4) \omega_{1}\right)}{\sin \left((N-3) \omega_{1}\right)} & 1 & 0 & 0 & \ldots & 0 & \frac{\sin \left(\omega_{1}\right)}{\sin \left((N-3) \omega_{1}\right)} \\
1 & -2 \cos \left(\omega_{1}\right) & 1 & 0 & \ldots & 0 & 0 \\
0 & 1 & -2 \cos \left(\omega_{1}\right) & 1 & \ldots & 0 & 0 \\
\vdots & \vdots & \vdots & \vdots & \vdots & \vdots & \vdots \\
0 & 0 & 0 & 0 & \ldots & -2 \cos \left(\omega_{1}\right) & 1 \\
\frac{\sin \left(\omega_{1}\right)}{\sin \left((N-3) \omega_{1}\right)} & 0 & 0 & 0 & \ldots & 1 & -\frac{\sin \left((N-4) \omega_{1}\right)}{\sin \left((N-3) \omega_{1}\right)}
\end{array}\right] .
$$




$$
\begin{aligned}
= & \frac{1}{2 \sigma_{q}^{2}}((N-2)(N-3) \\
& \left.-\sum_{k=1}^{N-2}\left(\frac{\sin \left((N-2) \omega_{1}\right) \cos \left((N-2 k-1) \omega_{1}\right)}{\sin \left(\omega_{1}\right)}-1\right)\right) \\
& \times\left(\frac{1}{4 \sin ^{2}\left(\omega_{1}\right)}\right) \\
= & \frac{1}{2 \sigma_{q}^{2}}\left((N-2)^{2}-\frac{\sin ^{2}\left((N-2) \omega_{1}\right)}{\sin ^{2}\left(\omega_{1}\right)}\right)\left(\frac{1}{4 \sin ^{2}\left(\omega_{1}\right)}\right)
\end{aligned}
$$

where tr denotes the trace operator. Similarly, it is shown that

$$
\begin{array}{r}
D_{1}=\frac{1}{2 \sigma_{q}^{2}}\left((N-2)(N-3) \cos \left(\omega_{1}\right)-\frac{\cos \left(\omega_{1}\right)-\cos \left((2 N-5) \omega_{1}\right)}{2 \sin ^{2}\left(\omega_{1}\right)}\right) \\
\times\left(\frac{1}{4 \sin ^{2}\left(\omega_{1}\right)}\right) \quad(\mathrm{C} .2)
\end{array}
$$

and

$$
\begin{aligned}
& D_{2}=\frac{1}{2 \sigma_{q}^{2}}\left((N-4)(N-6) \cos \left(2 \omega_{1}\right)+(N-4)\left(\frac{\sin \left(\omega_{1}\right)+\sin \left(3 \omega_{1}\right)}{\sin \left(\omega_{1}\right)}\right)\right. \\
& \left.-\frac{\sin \left((N-2) \omega_{1}\right) \sin \left((N-4) \omega_{1}\right)}{\sin ^{2}\left(\omega_{1}\right)}\right) \cdot\left(\frac{1}{4 \sin ^{2}\left(\omega_{1}\right)}\right) \cdot \quad \text { (C.3) }
\end{aligned}
$$

From (C.1)-(C.3), we have

$$
\lim _{N \rightarrow \infty} \frac{D_{1}}{D_{0}}=\cos \left(\omega_{1}\right)
$$

and

$$
\lim _{N \rightarrow \infty} \frac{D_{2}}{D_{0}}=\cos \left(2 \omega_{1}\right) .
$$

Substituting (C.4) and (C.5) into (32) yields (35).

\section{APPENDIX D}

We first derive the bias of $\hat{a}_{1}$ and then its variance for sufficiently large $N$. The values of $E\left\{\mathbf{X}_{\mathbf{2}}^{T} \cdot \mathbf{U}^{2} \cdot\left(\mathbf{Q}_{\mathbf{1}}+a_{1} \mathbf{Q}_{\mathbf{2}}\right)\right\}$ and $E\left\{\mathbf{X}_{\mathbf{2}}^{T} \cdot \mathbf{U}^{2} \cdot \mathbf{X}_{\mathbf{2}}\right\}$ in (41) are determined as follows. The term $E\left\{\mathbf{X}_{\mathbf{2}}{ }^{T} \cdot \mathbf{U}^{2} \cdot\left(\mathbf{Q}_{\mathbf{1}}+a_{1} \mathbf{Q}_{\mathbf{2}}\right)\right\}$ can be written as

$$
\begin{aligned}
E\left\{\mathbf{X}_{\mathbf{2}}{ }^{T} \cdot \mathbf{U}^{2} \cdot\left(\mathbf{Q}_{\mathbf{1}}+a_{1} \mathbf{Q}_{\mathbf{2}}\right)\right\} & =E\left\{\operatorname{tr}\left(\mathbf{X}_{\mathbf{2}}{ }^{T} \cdot \mathbf{U}^{2} \cdot\left(\mathbf{Q}_{\mathbf{1}}+a_{1} \mathbf{Q}_{\mathbf{2}}\right)\right)\right\} \\
& =\operatorname{tr}\left(\mathbf{U}^{2} \cdot E\left\{\left(\mathbf{Q}_{\mathbf{1}}+a_{1} \mathbf{Q}_{\mathbf{2}}\right) \cdot \mathbf{X}_{\mathbf{2}}{ }^{T}\right\}\right) .
\end{aligned}
$$

The term $E\left\{\left(\mathbf{Q}_{\mathbf{1}}+a_{1} \mathbf{Q}_{\mathbf{2}}\right) \cdot \mathbf{X}_{\mathbf{2}}{ }^{T}\right\}$ is computed as

$$
E\left\{\left(\mathbf{Q}_{\mathbf{1}}+a_{1} \mathbf{Q}_{\mathbf{2}}\right) \cdot \mathbf{X}_{\mathbf{2}}{ }^{T}\right\}=\left[\begin{array}{ccccccc}
a_{1} & 1 & 0 & 0 & 0 & \cdots & 0 \\
1 & a_{1} & 1 & 0 & 0 & \cdots & 0 \\
0 & 1 & a_{1} & 1 & 0 & \cdots & 0 \\
\vdots & \vdots & \vdots & \vdots & \vdots & \vdots & \vdots \\
0 & 0 & 0 & 0 & 0 & \cdots & a_{1}
\end{array}\right] \sigma_{q}^{2} .
$$

Substituting (D.2) into (D.1) and using (C.1) and (C.2), we get

$$
\begin{aligned}
\operatorname{tr} & \left(\mathbf{U}^{2} \cdot E\left\{\left(\mathbf{Q}_{\mathbf{1}}+a_{1} \mathbf{Q}_{\mathbf{2}}\right) \cdot \mathbf{X}_{\mathbf{2}}^{T}\right\}\right) \\
= & \left(a_{1} D_{0}+2 D_{1}\right) \sigma_{q}^{2} \\
= & \left(-2 \cos \left(\omega_{1}\right) D_{0}+2 D_{1}\right) \sigma_{q}^{2} \\
= & \left(-(N-2) \cos \left(\omega_{1}\right)+\frac{\cos \left(\omega_{1}\right) \sin ^{2}\left((N-2) \omega_{1}\right)}{\sin ^{2}\left(\omega_{1}\right)}\right. \\
& \left.\quad-\frac{\cos \left(\omega_{1}\right)-\cos \left((2 N-5) \omega_{1}\right)}{2 \sin ^{2}\left(\omega_{1}\right)}\right)\left(\frac{1}{4 \sin ^{2}\left(\omega_{1}\right)}\right) \sigma_{q}^{2} .
\end{aligned}
$$

On the other hand, the term $E\left\{\mathbf{X}_{\mathbf{2}}^{T} \cdot \mathbf{U}^{2} \cdot \mathbf{X}_{\mathbf{2}}\right\}$ can be expressed as

$$
E\left\{\mathbf{X}_{\mathbf{2}}^{T} \cdot \mathbf{U}^{2} \cdot \mathbf{X}_{\mathbf{2}}\right\}=\mathbf{S}_{\mathbf{2}}{ }^{T} \cdot \mathbf{U}^{2} \cdot \mathbf{S}_{\mathbf{2}}+\sigma_{q}^{2} \operatorname{tr}\left(\mathbf{U}^{2}\right) .
$$

Using (C.1) again, we have

$\operatorname{tr}\left(\mathbf{U}^{2}\right)=\frac{1}{2}\left((N-2)^{2}-\frac{\sin ^{2}\left((N-2) \omega_{1}\right)}{\sin ^{2}\left(\omega_{1}\right)}\right)\left(\frac{1}{4 \sin ^{2}\left(\omega_{1}\right)}\right)$.

To compute $\mathbf{S}_{\mathbf{2}}{ }^{T} \cdot \mathbf{U}^{2} \cdot \mathbf{S}_{\mathbf{2}}$, we first examine the vector $\mathbf{S}_{\mathbf{2}}{ }^{T} \cdot \mathbf{U}$, whose $k$ th element is evaluated as (D.6), shown at the top of the next page, for sufficiently large $N$. Using (D.6) and considering that $N$ is large enough, we get

$$
\begin{aligned}
& \mathbf{S}_{\mathbf{2}}{ }^{T} \cdot \mathbf{U}^{2} \cdot \mathbf{S}_{\mathbf{2}} \\
& =\left(\mathbf{S}_{\mathbf{2}}{ }^{T} \cdot \mathbf{U}\right) \cdot\left(\mathbf{S}_{\mathbf{2}}{ }^{T} \cdot \mathbf{U}\right)^{T} \\
& \approx \frac{\alpha_{1}^{2}}{16 \sin ^{2}\left(\omega_{1}\right)} \sum_{k=1}^{N-1}\left((N-2 k-1)^{2} \sin ^{2}\left((N-k-2) \omega_{1}+\phi_{1}\right)\right) \\
& =\frac{\alpha_{1}^{2}}{32 \sin ^{2}\left(\omega_{1}\right)} \sum_{k=1}^{N-2}\left((N-2 k-1)^{2}\left(1-\cos \left(2(N-k-2) \omega_{1}+2 \phi_{1}\right)\right)\right) \\
& \approx \frac{\alpha_{1}^{2}}{32 \sin ^{2}\left(\omega_{1}\right)} \sum_{k=1}^{N-2}(N-2 k-1)^{2} \\
& =\frac{\alpha_{1}^{2}(N-2)\left((N-2)^{2}-1\right)}{96 \sin ^{2}\left(\omega_{1}\right)} .
\end{aligned}
$$

Substituting (D.3)-(D.5) and (D.7) into (41), and considering $N \rightarrow \infty$ gives (42), note that since $\mathbf{S}_{\mathbf{2}}{ }^{T} \cdot \mathbf{U}^{2} \cdot \mathbf{S}_{\mathbf{2}}$ and $\operatorname{tr}\left(\mathbf{U}^{2}\right)$ are of $O\left(N^{3}\right)$ and $O\left(N^{2}\right), E\left\{\mathbf{X}_{\mathbf{2}}{ }^{T} \cdot \mathbf{U}^{2} \cdot \mathbf{X}_{\mathbf{2}}\right\} \approx \mathbf{S}_{\mathbf{2}}{ }^{T} \cdot \mathbf{U}^{2} \cdot \mathbf{S}_{\mathbf{2}}$ for sufficiently large $N$, and thus, the expectation approximation made in (41) is valid.

The derivation of (43) is now given as follows. From (40), we have

$$
\begin{aligned}
& \left(\hat{a}_{1}-a_{1}\right)^{2} \\
& =\left(\mathbf{X}_{\mathbf{2}}{ }^{T} \cdot \mathbf{W} \cdot \mathbf{X}_{\mathbf{2}}\right)^{-1} \cdot \mathbf{X}_{\mathbf{2}}{ }^{T} \cdot \mathbf{W} \cdot\left(\mathbf{Q}_{\mathbf{1}}+a_{1} \mathbf{Q}_{\mathbf{2}}\right) \\
& \quad \cdot\left(\mathbf{Q}_{\mathbf{1}}+a_{1} \mathbf{Q}_{\mathbf{2}}\right)^{T} \cdot \mathbf{W} \cdot \mathbf{X}_{\mathbf{2}} \cdot\left(\mathbf{X}_{\mathbf{2}}{ }^{T} \cdot \mathbf{W} \cdot \mathbf{X}_{\mathbf{2}}\right)^{-1}(\mathrm{D} .8)
\end{aligned}
$$

because $\left(\mathbf{X}_{\mathbf{2}}^{T} \cdot \mathbf{W} \cdot \mathbf{X}_{\mathbf{2}}\right)^{-1}$ and $\mathbf{W}$ are symmetric. It is seen that the expected value of (D.8) is difficult to compute. How- 


$$
\begin{aligned}
\frac{\alpha_{1}}{2 \sin \left(\omega_{1}\right)} & \sum_{i=1}^{N-k-2} \sin \left(i \omega_{1}\right) \cos \left((N-k-i-1) \omega_{1}+\phi_{1}\right)+\alpha_{1} \sum_{i=1}^{k-1} \sin \left(i \omega_{1}\right) \cos \left((N-k+i-1) \omega_{1}+\phi_{1}\right) \\
= & \frac{\alpha_{1}}{4 \sin \left(\omega_{1}\right)}\left[\sum_{i=1}^{N-k-2}\left(\sin \left((N-k-1) \omega_{1}+\phi_{1}\right)-\sin \left((N-k-2 i-1) \omega_{1}+\phi_{1}\right)\right]\right] \\
& +\frac{\alpha_{1}}{4 \sin \left(\omega_{1}\right)}\left[\sum_{i=1}^{k-1}\left(\sin \left((N-k+2 i-1) \omega_{1}+\phi_{1}\right)-\sin \left((N-k-1) \omega_{1}+\phi_{1}\right)\right]\right] \\
= & \frac{\alpha_{1}}{4 \sin \left(\omega_{1}\right)}\left[(N-k-2) \sin \left((N-k-1) \omega_{1}+\phi_{1}\right)-(k-1) \sin \left((N-k-1) \omega_{1}+\phi_{1}\right)\right] \\
& +\frac{\alpha_{1}}{4 \sin \left(\omega_{1}\right)}\left[\frac{\sin \left((k-1) \omega_{1}\right) \sin \left((N-1) \omega_{1}+\phi_{1}\right)}{\sin \left(\omega_{1}\right)}-\frac{\sin \left(\phi_{1}\right) \sin \left((N-k-2) \omega_{1}\right)}{\sin \left(\omega_{1}\right)}\right] \\
\approx & \frac{\alpha_{1}}{4 \sin \left(\omega_{1}\right)}(N-2 k-1) \sin \left((N-k-1) \omega_{1}+\phi_{1}\right), \quad k=1,2, \ldots, N-2
\end{aligned}
$$

ever, the analysis can be much simplified if the approximation of $E\left\{\mathbf{X}_{\mathbf{2}}^{T} \cdot \mathbf{W} \cdot \mathbf{X}_{\mathbf{2}}\right\} \approx \sigma_{q}^{-2} \mathbf{S}_{\mathbf{2}}^{T} \cdot \mathbf{U}^{2} \cdot \mathbf{S}_{\mathbf{2}} \approx \mathbf{S}_{\mathbf{2}}^{T} \cdot \mathbf{W} \cdot \mathbf{S}_{\mathbf{2}}$, and the law of iterated expectations are employed. With the use of (D.4), (D.5), and (D.7), $\operatorname{var}\left(\hat{a}_{1}\right)$ is derived as follows:

$$
\begin{aligned}
\operatorname{var}\left(\hat{a}_{1}\right)= & E\left\{\left(\hat{a}_{1}-a_{1}\right)^{2}\right\} \\
\approx & \left(\mathbf{S}_{\mathbf{2}}{ }^{T} \cdot \mathbf{W} \cdot \mathbf{S}_{\mathbf{2}}\right)^{-1} \cdot E\left\{\mathbf{X}_{\mathbf{2}}{ }^{T} \cdot \mathbf{W} \cdot\left(\mathbf{Q}_{\mathbf{1}}+a_{1} \mathbf{Q}_{\mathbf{2}}\right)\right. \\
& \left.\cdot\left(\mathbf{Q}_{\mathbf{1}}+a_{1} \mathbf{Q}_{\mathbf{2}}\right)^{T} \cdot \mathbf{W} \cdot \mathbf{X}_{\mathbf{2}}\right\} \cdot\left(\mathbf{S}_{\mathbf{2}}{ }^{T} \cdot \mathbf{W} \cdot \mathbf{S}_{\mathbf{2}}\right)^{-1} \\
= & \left(\mathbf{S}_{\mathbf{2}}{ }^{T} \cdot \mathbf{W} \cdot \mathbf{S}_{\mathbf{2}}\right)^{-1} \cdot E\left\{\mathbf { X } _ { \mathbf { 2 } } { } ^ { T } \cdot \mathbf { W } \cdot E \left\{\left(\mathbf{Q}_{\mathbf{1}}+a_{1} \mathbf{Q}_{\mathbf{2}}\right)\right.\right. \\
& \left.\left.\cdot\left(\mathbf{Q}_{\mathbf{1}}+a_{1} \mathbf{Q}_{\mathbf{2}}\right)^{T}\right\} \cdot \mathbf{W} \cdot \mathbf{X}_{\mathbf{2}}\right\} \\
& \cdot\left(\mathbf{S}_{\mathbf{2}}{ }^{T} \cdot \mathbf{W} \cdot \mathbf{S}_{\mathbf{2}}\right)^{-1} \\
= & \left(\mathbf{S}_{\mathbf{2}}{ }^{T} \cdot \mathbf{W} \cdot \mathbf{S}_{\mathbf{2}}\right)^{-1} \cdot E\left\{\mathbf{X}_{\mathbf{2}}{ }^{T} \cdot \mathbf{W} \cdot \mathbf{W}^{-1} \cdot \mathbf{W} \cdot \mathbf{X}_{\mathbf{2}}\right\} \\
& \cdot\left(\mathbf{S}_{\mathbf{2}}{ }^{T} \cdot \mathbf{W} \cdot \mathbf{S}_{\mathbf{2}}\right)^{-1} \\
\approx & \left(\mathbf{S}_{\mathbf{2}}{ }^{T} \cdot \mathbf{W} \cdot \mathbf{S}_{\mathbf{2}}\right)^{-1} \\
\approx & \frac{\sigma_{q}^{2}}{\mathbf{S}_{\mathbf{2}}{ }^{T} \cdot \mathbf{U}^{2} \cdot \mathbf{S}_{\mathbf{2}}} \\
\approx & \frac{48 \sin ^{2}\left(\omega_{1}\right)}{\operatorname{SNR}(N-2)\left((N-2)^{2}-1\right)}
\end{aligned}
$$

which is (43).

\section{ACKNOWLEDGMENT}

The authors wish to thank the anonymous reviewers for their careful reading and valuable comments that improved the quality of this paper.

\section{REFERENCES}

[1] P. Stoica, "List of references on spectral line analysis," Signal Process., vol. 31, no. 3, pp. 329-340, Apr. 1993.

[2] S. L. Marple, Digital Spectral Analysis with Applications. Englewood Cliffs, NJ: Prentice-Hall, 1987.

[3] S. M. Kay, Modern Spectral Estimation: Theory and Application. Englewood Cliffs, NJ: Prentice-Hall, 1988.

[4] B. Porat, Digital Processing of Random Signals: Theory and Methods. Englewood Cliffs, NJ: Prentice-Hall, 1994.
[5] P. Stoica and R. Moses, Introduction to Spectral Analysis. Upper Saddle River, NJ: Prentice-Hall, 1997.

[6] B. G. Quinn and E. J. Hannan, The Estimation and Tracking of Frequency. Cambridge, U.K.: Cambridge Univ. Press, 2001.

[7] D. C. Rife and R. R. Boorstyn, "Multiple tone parameter estimation from discrete-time observations," Bell Syst. Tech. J., pp. 1389-1410, Nov. 1976.

[8] P. Stoica and A. Nehorai, "Statistical analysis of two nonlinear leastsquares estimators of sine wave parameters in the colored noise case," in Proc. Int. Conf. Acoust. Speech, Signal Processing, vol. 4, New York, 1988, pp. 2408-2411.

[9] Y. T. Chan, J. M. M. Lavoie, and J. B. Plant, "A parameter estimation approach to estimation of frequencies of sinusoids," IEEE Trans. Acoust. Speech, Signal Process., vol. ASSP-29, no. 2, pp. 214-219, Apr. 1981.

[10] Y. T. Chan and R. P. Langford, "Spectral estimation via the high-order Yule-Walker equations," IEEE Trans. Acoust. Speech, Signal Processing, vol. ASSP-30, no. 5, pp. 689-698, Oct. 1982.

[11] M. D. Rahman and K. B. Yu, "Total least squares approach for frequency estimation using linear prediction," IEEE Trans. Acoust. Speech, Signal Processing, vol. ASSP-35, no. 5, pp. 1440-1454, Oct. 1987.

[12] T. H. Li and B. Kedem, "Iterative filtering for multiple frequency estimation," IEEE Trans. Signal Processing, vol. 42, no. 5, pp. 1120-1131, May 1994.

[13] H. C. So and K. W. Chan, "Reformulation of Pisarenko harmonic decomposition method for single-tone frequency estimation," IEEE Trans. Signal Processing, vol. 52, no. 4, pp. 1128-1135, Apr. 2004.

[14] V. F. Pisarenko, "The retrieval of harmonics by linear prediction," Geophys. J. Roy. Astron. Soc., vol. 33, pp. 347-366, 1973.

[15] M. V. Dragosevic and S. S. Stankovic, "A generalized least squares method for frequency estimation," IEEE Trans. Acoust., Speech, Signal Process., vol. 37, no. 6, pp. 805-819, Jun. 1989.

[16] S. M. Kay and V. Nagesha, "Spectral analysis based on the canonical autoregressive decomposition," in Proc. Int. Conf. Acoust., Speech, Signal Process., vol. 5, Toronto, ON, Canada, Apr. 1991, pp. 3137-3140.

[17] X.-D. Zhang, Y.-C. Liang, and Y.-D. Li, "A hybrid approach to harmonic retrieval in non-Gaussian ARMA noise," IEEE Trans. Inf. Theory, vol. 40, no. 4, pp. 1220-1226, Jul. 1994.

[18] C.-M. Cho and P. M. Djuric, "Bayesian detection and estimation of cisoids in colored noise," IEEE Trans. Signal Process., vol. 43, no. 12, pp. 2943-2952, Dec. 1995.

[19] Z.-S. Liu and J. Li, "Implementation of the RELAX algorithm," IEEE Trans. Aerosp. Electron. Syst., vol. 34, no. 4, pp. 657-664, Apr. 1998.

[20] P. A. Thompson, "An adaptive spectral analysis technique for unbiased frequency estimation in the presence of white noise," in Proc. 13th Asilomar Conf. Circuits, Syst., Comput., Pacific Grove, CA, Nov. 1979, pp. 529-533.

[21] P. A. Regalia, "An unbiased equation error identifier and reduced-order approximations," IEEE Trans. Signal Process., vol. 42, no. 6, pp. 1397-1412, Jun. 1994.

[22] T. K. Moon and W. C. Stirling, Mathematical Methods and Algorithms for Signal Processing. Upper Saddle River, NJ: Prentice-Hall, 2000. 
[23] T. Soderstrom and P. Stoica, System Identification. Englewood Cliffs, NJ: Prentice-Hall, 1989.

[24] G. C. Goodwin and R. L. Payne, Dynamic System Identification: Experiment Design and Data Analysis. New York: Academic, 1977.

[25] H. N. Kim and W. J. Song, "Unbiased equation-error adaptive IIR filtering based on monic normalization," IEEE Signal Process. Lett., vol. 6, no. 2, pp. 35-37, Feb. 1999.

[26] R. Lopez-Valcarce and S. Dasgupta, "A new proof for the stability of equation-error models," IEEE Signal Process. Lett., vol. 6, no. 6, pp. 148-150, Jun. 1999.

[27] R. Kumaresan, L. L. Scharf, and A. K. Shaw, "An algorithm for pole-zero modeling and spectral analysis," IEEE Trans. Acoust., Speech, Signal Process., vol. ASSP-34, no. 3, pp. 637-640, Jun. 1986.

[28] Y. Bresler and A. Macovski, "Exact maximum likelihood parameter estimation of superimposed exponential signals in noise," IEEE Trans. Acoust., Speech, Signal Processing, vol. 34, pp. 1081-1089, Oct. 1986.

[29] K. W. Chan and H. C. So, "Accurate frequency estimation for real harmonic sinusoids," IEEE Signal Process. Lett., vol. 11, no. 7, pp. 609-612, Jul. 2004.

[30] F. A. Graybill, Matrices with Applications in Statistics. Belmont, CA: Wadsworth, 1983, p. 201.

[31] M. J. R. Healy, Matrices for Statistics. New York: Oxford Univ. Press, 2000, pp. 41-42.

[32] B. Noble and J. W. Daniel, Applied Linear Algebra. Englewood Cliffs, NJ: Prentice-Hall, 1988.

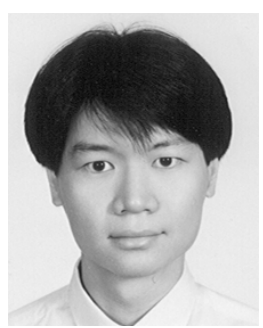

H. C. So (M'95) was born in Hong Kong. He received the B.Eng. degree in electronic engineering from City Polytechnic of Hong Kong in 1990 and the Ph.D. degree in electronic engineering from The Chinese University of Hong Kong in 1995

From 1990 to 1991, he was an Electronic Engineer with the Research and Development Division, Everex Systems Engineering Ltd., Hong Kong. During 1995 and 1996, he was a Post-Doctoral Fellow at The Chinese University of Hong Kong and was responsible for devising and analyzing efficient algorithms for geolocation. From 1996 to 1999, he was a Research Assistant Professor with the Department of Electronic Engineering, City University of Hong Kong. Currently, he is an Associate Professor with the Department of Computer Engineering and Information Technology, City University of Hong Kong. His research interests include adaptive filter theory, detection and estimation, wavelet transform, and signal processing for communications and multimedia.

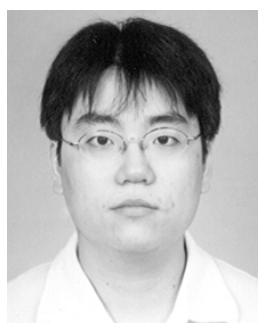

Kit Wing Chan received the B.Eng. degree in computer engineering and the M.Phil degree from City University of Hong Kong in 2002 and 2005, respectively.

His research interests include statistical signal processing and their applications, with particular attention to frequency estimation and related mathematics.

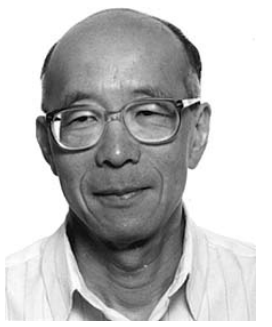

Y. T. Chan (SM'80) was born in Hong Kong and received his electrical engineering education in Canada. He received the Bachelor's and Master's degrees from Queen's University, Kingston, ON, Canada, the Ph.D. degree from the University of New Brunswick, Fredericton.

$\mathrm{He}$ was an engineer with Nortel Networks, London, ON, and Ottawa, ON, and has been a professor with the Electrical and Computer Engineering Department, Royal Military College of Canada, Kingston, serving as head of the Department from 1994 to 2000. He joined the Electronic Engineering Department, The Chinese University of Hong Kong, in 2001 as a visiting professor. Presently, he is an adjunct professor with the Royal Military College of Canada. His research interests are in detection, estimation, localization, and tracking. He has published the textbook Wavelet Basics (Boston, MA: Kluwer, 1994).

Dr. Chan was an associate editor of the IEEE TRANSACTIONS ON SIGNAL PROCESSING, the technical chair of ICASSP-84, general chair of ICASSP-91, vice-chair of ICASSP-03, and social chair of ICASSP-04. He directed a NATO ASI in 1988

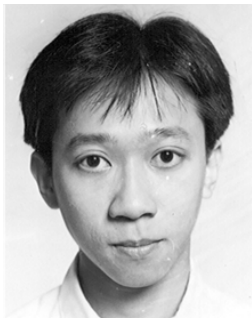

K. C. Ho (' $\left.89-\mathrm{M}^{\prime} 91-\mathrm{SM}^{\prime} 00\right)$ was born in Hong Kong. He received the B.Sc. degree with First Class Honors in electronics and the Ph.D. degree in electronic engineering from the Chinese University of Hong Kong, in 1988 and 1991, respectively.

He was a research associate with the Department of Electrical and Computer Engineering, the Royal Military College of Canada, Kingston, ON, Canada, from 1991 to 1994. He joined the Bell-Northern Research, Montreal, QC, Canada, in 1995 as a member of scientific staff. He was a faculty member with the Department of Electrical Engineering, the University of Saskatchewan, Saskatoon, SK, Canada, from September 1996 to August 1997. Since September 1997, he has been with the University of Missouri-Columbia, where he is currently an Associate Professor with the Electrical and Computer Engineering Department. He is also an Adjunct Associate Professor at the Royal Military College of Canada. His research interests are in detection and estimation, source localization, wavelet transform, wireless communications, subsurface object detection, and the development of efficient adaptive signal processing algorithms for various applications including landmine detection, echo cancellation, and time delay estimation. He has been active in the development of the ITU Standard Recommendation G.168: Digital Network Echo Cancellers since 1995. He is the editor of the ITU Standard Recommendation G.168. He has three patents from the United States in the area of telecommunications.

Dr. Ho is currently an Associate Editor of the IEEE TRANSACTIONS ON Signal Processing and the IEEE Signal Processing LetTers. He was the recipient of the Croucher Foundation Studentship from 1988 to $1991 . \mathrm{He}$ received the Junior Faculty Research Award from College of Engineering, University of Missouri-Columbia, in 2003. 